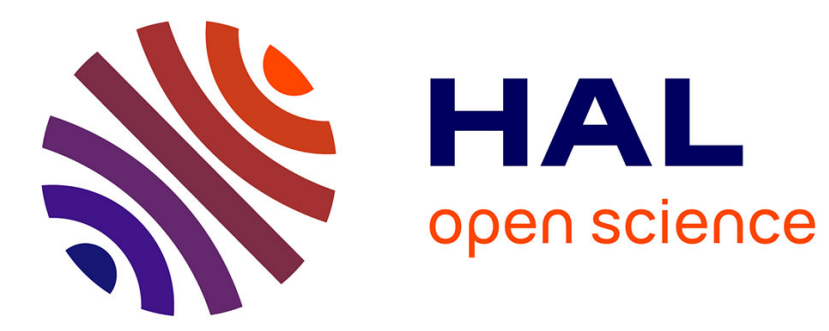

\title{
Homology stability for unitary groups over S-arithmetic rings \\ Gaël Collinet
}

\section{- To cite this version:}

Gaël Collinet. Homology stability for unitary groups over S-arithmetic rings. Journal of K-theory , 2010, pp.30. 10.1017/is010010004jkt123 . hal-00574020

\section{HAL Id: hal-00574020 \\ https://hal.science/hal-00574020}

Submitted on 7 Mar 2013

HAL is a multi-disciplinary open access archive for the deposit and dissemination of scientific research documents, whether they are published or not. The documents may come from teaching and research institutions in France or abroad, or from public or private research centers.
L'archive ouverte pluridisciplinaire HAL, est destinée au dépôt et à la diffusion de documents scientifiques de niveau recherche, publiés ou non, émanant des établissements d'enseignement et de recherche français ou étrangers, des laboratoires publics ou privés. 


\title{
HOMOLOGY STABILITY FOR UNITARY GROUPS OVER $S$-ARITHMETIC RINGS
}

\author{
G. COLLINET
}

\begin{abstract}
We prove that the homology of unitary groups over rings of $S$-integers in number fields stabilizes. Results of this kind are well known to follow from the high acyclicity of $a d-h o c$ polyhedra. Given this, we exhibit two simple conditions on the arithmetic of hermitian forms over a ring $A$ relatively to an antiautomorphism which, if they are satisfied, imply the stabilization of the homology of the corresponding unitary groups. When $R$ is a ring of $S$-integers in a number field $K$, and $A$ is a maximal $R$-order in an associative composition algebra $F$ over $K$, we use the strong approximation theorem to show that both of these properties are satisfied. Finally we take a closer look at the case of $\mathrm{O}_{n}\left(\mathbb{Z}\left[\frac{1}{2}\right]\right)$.
\end{abstract}

\section{INTRODUCTION}

- The result in the general case.

Let $R$ be a commutative ring with unit, and $C$ an associative $R$-algebra together with an anti-automorphism $c \mapsto \bar{c}$ centralizing $R$. An h-module over $\mathrm{C}$ is a pair $\mathrm{M}=(M, h)$ consisting of a finitely generated projective left $C$-module $M$ and a hermitian form $h: M \times M \rightarrow C$ (i.e. $h(y, x)=\overline{h(x, y)}$ and $h(a x, b y)=a h(x, y) \bar{b})$ that is non-degenerate. When $\mathrm{M}$ is an h-module over $C$ we denote by $\mathrm{U}(\mathrm{M})$ the group of automorphisms of $\mathrm{M}$ and when $\mathrm{M}$ and $\mathrm{N}$ are two h-modules over $C$, and $n \geq 0$ is an integer we denote by $\mathrm{M}^{\perp} \mathrm{N}^{\perp n}$ the orthogonal sum of one copy of $\mathrm{M}$ and $n$ copies of $\mathrm{N}$, and $i(\mathrm{M}, \mathrm{N}, n)$ the natural inclusion

$$
\mathrm{U}\left(\mathrm{M} \perp \mathrm{N}^{\perp n}\right) \rightarrow \mathrm{U}\left(\mathrm{M} \perp \mathrm{N}^{\perp n} \perp \mathrm{N}\right)=\mathrm{U}\left(\mathrm{M} \perp \mathrm{N}^{\perp n+1}\right) .
$$

Our aim in this work is to give a proof of the following

Theorem. Let $K$ be a global field of characteristic 0 and let $\mathrm{C}_{K}$ be an associative composition algebra over $K$ together with its canonical anti-automorphism. Let $\Omega$ be a finite subset of the set $\mathcal{V}$ of all places of $K$, containing all archimedean places and at least one non-archimedean place and let $S$ be its complement in $\mathcal{V}$. Let $A$ be the ring of $S$-integers in $K$, and let $\mathrm{C}$ be a maximal A-order in $\mathrm{C}_{K}$. Let $\mathrm{M}$ and $\mathrm{N}$ be $\mathrm{h}$-modules over $\mathrm{C}$ (resp. $\mathrm{C}_{K}$ ) of respective ranks $\mu$ and $\nu$.

There exists two integers $\alpha \leq 16+64 \nu$ and $\beta \leq 69+64 \nu$ such that the morphism $\mathrm{H}_{k}\left(\mathrm{U}\left(\mathrm{M} \perp \mathrm{N}^{n}\right) ; \mathbb{Z}\right) \rightarrow$ $\mathrm{H}_{k}\left(\mathrm{U}\left(\mathrm{M} \perp \mathrm{N}^{n+1}\right) ; \mathbb{Z}\right)$ induced in $k$-dimensional homology by $i(\mathrm{M}, \mathrm{N}, n)$ is an isomorphism for $n \geq \alpha k+\beta$ and an epimorphism for $n+\alpha \geq \alpha k+\beta$.

- Examples.

Let $N \geq 2$ be an integer and take $A=\mathbb{Z}\left[\frac{1}{N}\right]$, the subring of $K=\mathbb{Q}$ whose elements are irreducible fractions with denominator dividing $N^{k}$ for $k$ large enough.

○ Taking $\mathrm{C}_{K}=K$ with trivial involution, $\mathrm{M}=0$ the trivial h-module and $\mathrm{N}=(A, h)$ defined by $h(x, y)=$ $x y$, the theorem says that the homology of $\mathrm{O}_{n}(A)$ stabilizes, where $\mathrm{O}_{n}(A)$ is the subgroup of the compact Lie group $\mathbf{O}_{n}$ of $n \times n$ orthogonal matrices with entries in $A$.

○ Taking $\mathrm{C}_{K}=K[i]$ with complex conjugation, $\mathrm{M}=0$ the trivial h-module and $\mathrm{N}=(A[i], h)$ defined by $h(x, y)=x \bar{y}$, the theorem says that the homology of $\mathrm{U}_{n}(A)$ stabilizes, where $\mathrm{U}_{n}(A)$ is the subgroup of the compact Lie group $\mathbf{U}_{n}$ of $n \times n$ unitary matrices with entries in $A[i]$.

2010 Mathematics Subject Classification. 11F75, 19B14, 51F25.

Key words and phrases. Homology stability, unitary groups, $S$-arithmetic groups, adjacency relations, strong approximation.

Partially supported by project ANR blanc BLAN08-2_338236, HGRT. The author also acknowledges support from the Swiss National Science Foundation. 
○ Taking $\mathrm{C}_{K}=K[1, i, j, i j]$, the standard Hamilton quaternion algebra with quaternionic conjugation, $\mathrm{M}=0$ the trivial h-module, $C=A\left[1, i, j, \frac{1+i+j+i j}{2}\right]$ and $\mathrm{N}=(C, h)$ defined by $h(x, y)=x \bar{y}$, the theorem says that the homology of $\operatorname{Sp}_{n}(A)$ stabilizes, where $\operatorname{Sp}_{n}(A)$ is the subgroup of the compact Lie group $\mathbf{S p}_{n}$ of $n \times n$ unitary matrices with entries in $C$.

○ Taking $\mathrm{C}_{K}=K \times K$ with $\overline{(a, b)}=(b, a), \mathrm{M}=0$ the trivial h-module and $\mathrm{N}=(A \times A, h)$ defined by $h(x, y)=x \bar{y}$, the theorem says that the homology of $\mathrm{GL}_{n}(A)$ stabilizes, where $\mathrm{GL}_{n}(A)$ is the subgroup of the rank $n$ Lie group $\mathbf{G L}_{n}(\mathbb{R})$ of $n \times n$ invertible matrices with entries in $A$.

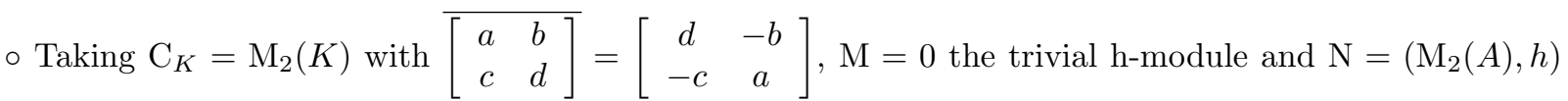
defined by $h(x, y)=x \bar{y}$, the theorem says that the homology of $\operatorname{Sp}_{2 n}^{s}(A)$ stabilizes, where $\operatorname{Sp}_{2 n}^{s}(A)$ is the subgroup of the rank $n$ Lie group $\mathbf{S p}_{2 n}^{s}(\mathbb{R})$ of $2 n \times 2 n$ symplectic matrices with entries in $A$.

- This result takes place in the large family of homology stability theorems for linear algebraic groups over nice rings, the genealogy of which may be traced back to the early seventies with the birth of D. Quillen's K-theory. Significant results were soon obtained by Quillen himself for $\mathrm{GL}_{n}$ over fields (unpublished), by K. Vogtmann in her thesis [V1977] for $\mathrm{O}_{n, n}$ over fields, by R. Charney [Ch1979] for $\mathrm{GL}_{n}$ over Dedekind domains, by H. Maazen and W. van der Kallen [vdK1980] for $\mathrm{GL}_{n}$ over rings satisfying one of the properties known under Bass's stable range conditions and by K. Vogtmann [V1982] for $\mathrm{O}_{n}$ over fields with finite pythagorean number. More recently, B. Mirzaii and van der Kallen [MvdK2002] proved stability for unitary groups of hyperbolic spaces over rings satisfying a unitary version of Bass's stable range conditions, and J.-L. Cathelineau [Ca2007] gave very sharp information for $\mathrm{O}_{n}$ over pythagorean fields.

- Reduction to two conditions on the arithmetic of h-modules over C.

The strategy to prove all these results is the same. We summarize it in the following theorem whose proof (essentially a spectral sequence argument) may be recovered from each of the references above (see also [HW $5.1])$.

Stability theorem. Let $\Gamma_{0} \subset \Gamma_{1} \subset \Gamma_{2} \subset \cdots \subset \Gamma_{s} \subset \ldots$ be an increasing sequence of groups, each of which acts on a triangulated space ${ }^{1} X_{s}$ of dimension at least $s-1$. Assume there exists an increasing sequence $f$ of integers $f_{k} \geq 2 k+1$ such that

(A): for all $k \geq 0$, the space $X_{s}$ is $k$-acyclic for all $s \geq f_{k}$ (i.e. $\tilde{\mathrm{H}}_{l}(X)$ is trivial for $\left.l \leq k\right)$.

Assume moreover that the action of $\Gamma_{s}$ on $X_{s}$ satisfies

(C): it is transitive on the set $\left(X_{s}\right)_{t}$ of $t$-dimensional simplices of $X_{s}$, for $t \leq s-1$,

$(\mathrm{R}):$ for $t \leq s-1$, the stabilizer $\Gamma_{s}(\sigma)$ of a $t$-dimensional simplex $\sigma$ is conjugate (in $\Gamma_{s}$ ) to $\Gamma_{s-t-1}$,

(S): an element of $\Gamma_{s}$ that stabilizes globally a simplex stabilizes it pointwise,

$(\mathrm{T})$ : for each 1-simplex $\sigma$ joining two 0-simplices a and $b$, there exists an element $\gamma$ of $\Gamma_{s}$ that commutes with $\Gamma_{s}(\sigma)$ such that the equalities $\gamma(a)=b$ and $\gamma(b)=a$ hold. (Note that property $(\mathrm{S})$ then implies $\gamma(\sigma) \neq \sigma$. )

Then for $s \geq f_{k}$, the inclusion of $\Gamma_{s-1}$ in $\Gamma_{s}$ induces an isomorphism (resp. an epimorphism) in l-dimensional homology for $l \leq k$ (resp. $l=k+1)$.

(Letters A and C stand respectively for the acyclicity condition and cancellability condition).

Let us come back to our situation: let $R$ be a ring, $C$ an associative $R$-algebra with anti-automorphism $c \mapsto \bar{c}$, and let $\mathrm{M}, \mathrm{N}$ be h-modules over $C$. We want to apply this theorem with $\Gamma_{s}=\mathrm{U}\left(\mathrm{M} \perp \mathrm{N}^{s+1}\right)$. In order to do so we need to introduce ad-hoc spaces $X_{s}$.

Let $\mathrm{P}=\left(P, h_{P}\right)$ be an h-module over $\mathrm{C}$, and let us call $h_{P}^{*}$ the restriction of $h_{P}$ to a given submodule of $P$. We introduce the set $\operatorname{Conf}(F, \mathrm{P})$ of linear isometries $\varphi: \mathrm{N} \rightarrow\left(\varphi(N), h_{P}^{*}\right) \subset \mathrm{P}$.

\footnotetext{
${ }^{1}$ In fact the geometric realization of a pre-simplicial set (see 1.1)
} 
This set comes with a natural adjacency (i.e. a symmetric anti-reflexive) relation $\perp$ defined by

$$
\varphi \perp \varphi^{\prime} \Leftrightarrow h_{P}\left(\varphi(n), \varphi^{\prime}\left(n^{\prime}\right)\right)=0 \quad \forall\left(n, n^{\prime}\right) \in \mathrm{N}^{2}
$$

To a set $E$ endowed with such an adjacency relation $\perp$ is naturally associated a geometric realization; roughly speaking, this is the triangulated space $|(E, \perp)|$ whose vertices are elements of $E$, and whose $k$ simplices are sequences $\left(x_{0}, \ldots, x_{k}\right)$ of mutually adjacent elements (see section 1 ).

We put $X_{s}=\left|\operatorname{Conf}\left(F, \mathrm{M} \perp \mathrm{N}^{s+1}\right), \perp\right|$. Clearly, in this situation, if condition (C) of the theorem holds, then so do conditions (R), (S) and (T). Thus we just need to check the acyclicity condition (A) and the cancellation condition $(\mathrm{C})$.

We shall give in section 1 a simple criterion to ensure that the geometric realization of a set $E$ endowed with an adjacency relation is highly acyclic: let us say that a sequence $\left(P_{1}, \ldots, P_{r}\right)$ of subsets of $E$ such that

$$
\operatorname{card}\left(P_{i}\right) \geq 2(\forall i), \quad P_{i} \perp P_{j}(\forall i \neq j), \quad \sum_{i=1}^{r}\left(\operatorname{card}\left(P_{i}\right)-1\right) \leq k+1
$$

is a $k$-test sequence.

Acyclicity criterion. Let $(E, \perp)$ be a set together with an adjacency relation. Assume for any $k$-test sequence $\left(P_{1}, \ldots, P_{r}\right)$ of subsets of $E$ the intersection $\cap_{i} P_{i}^{\perp}$ is non-empty. Then $|(E, \perp)|$ is $k$-acyclic.

(In particular, if for any sequence $\left(x_{1}, \ldots, x_{2 k+2}\right)$ of elements of $E$ there exists an element $y$ adjacent to each one of the $x_{i}$ 's, then $|(E, \perp)|$ is $k$-acyclic.)

Now consider the set $\operatorname{Rep}(\mathrm{N}, \mathrm{P})$ of representations of $\mathrm{N}$ by $\mathrm{P}$, i.e. the set of submodules $N^{\prime}$ of $P$ such that there exist an isomorphism $\mathrm{N} \simeq\left(N^{\prime}, h_{P}^{*}\right)$, together with the natural adjacency relation $\perp$ given by orthogonality. Of course, if the criterion is satisfied for $(\operatorname{Rep}(\mathrm{N}, \mathrm{P}), \perp)$, then it is satisfied for $(\operatorname{Conf}(F, \mathrm{P}), \perp)$. Thus for two hermitian modules $\mathrm{M}$ and $\mathrm{N}$ over $\mathrm{C}$, and for a sequence $f=\left(f_{k}\right)_{k \geq 0}$ of integers such that $f_{k} \geq 2 k+1$, we introduce the following properties:

$\mathcal{A}(\mathrm{M}, \mathrm{N}, f)$ : For all $n \geq f_{k}$, for all $k$-test sequences $\left(P_{1}, \ldots, P_{s}\right)$ of $\operatorname{Rep}\left(\mathrm{N}, \mathrm{M} \perp \mathrm{N}^{n+1}\right)$ there exists an element $\mathrm{T}$ in $\operatorname{Rep}\left(\mathrm{N}, \mathrm{M} \perp \mathrm{N}^{n+1}\right)$ such that $\mathrm{T}$ is orthogonal to all elements of each $P_{i}$.

$\mathcal{C}(\mathrm{M}, \mathrm{N})$ : Let $\mathrm{B}$ be an h-module over $\mathrm{C}$. The existence of an isomorphism $\mathrm{B} \perp \mathrm{N} \simeq \mathrm{M} \perp \mathrm{N}^{k} \perp \mathrm{N}(k \in \mathbb{N})$ implies the existence of an isomorphism $\mathrm{B} \simeq \mathrm{M} \perp \mathrm{N}^{k}$.

When both of these conditions are satisfied, we can use the stability theorem. Indeed $\mathcal{A}(\mathrm{M}, \mathrm{N}, f)$ (together with the acyclicity criterion) implies that condition (A) holds, and $\mathcal{C}(\mathrm{M}, \mathrm{N})$ implies that property $(\mathrm{C})$ holds.

For a sequence $f=\left(f_{k}\right)_{k \geq 0}$ of integers, let us say property $\mathrm{P}(\mathrm{M}, \mathrm{N}, f)$ holds if for all $k \geq 0$ and for all $n \geq f_{k}$, the morphism induced in $l$-dimensional homology by the inclusion $i(\mathrm{M}, \mathrm{N}, n)$

$$
\mathrm{H}_{l}\left(\mathrm{U}\left(\mathrm{M} \perp \mathrm{N}^{n}\right) ; \mathbb{Z}\right) \rightarrow \mathrm{H}_{l}\left(\mathrm{U}\left(\mathrm{N} \perp \mathrm{N}^{n+1}\right) ; \mathbb{Z}\right)
$$

is an epimorphism (resp. an isomorphism) for $l \leq k+1$ (resp. for $l \leq k$ ). The following proposition summarizes the above discussion.

Proposition. Let $R$ be a ring, let $C$ be an associative $R$-algebra with an anti-automorphism centralizing $R$, and let $\mathrm{M}$ and $\mathrm{N}$ be h-modules over $C$. Assume both conditions $\mathcal{A}(\mathrm{M}, \mathrm{N}, f)$ and $\mathcal{C}(\mathrm{M}, \mathrm{N})$ hold. Then property $\mathrm{P}(\mathrm{M}, \mathrm{N}, f)$ holds.

- Dealing with the two conditions when $C$ is one of the algebras considered in the theorem.

Thus the problem is reduced to the verification of properties $\mathcal{A}$ and $\mathcal{C}$.

In the case of a field where 2 is invertible, the cancellation property $\mathcal{C}$ is implied by Witt's theorem, so only the acyclicity property $\mathcal{A}$ needs to be verified. The latter is shown to hold for any $\mathrm{M}$ and $\mathrm{N}$ over local fields as a consequence of the fact that a 4-dimensional quadratic space over such a field is universal, and is easily checked for archimedean fields. The case of global fields follows by the Hasse principle (see subsection $2.2)$. 
In the case of a local ring $R$, the cancellability condition holds when $R$ is non-dyadic, but fails to hold in general when $R$ is dyadic. Nevertheless, $\mathcal{C}(\mathrm{M}, \mathrm{N})$ is satisfied for all $\mathrm{M}$ when $\mathrm{N}$ is a sum of hyperbolic planes, so we restrict, when necessary, our attention to hyperbolic N's (see 2.4) during the verification of property $\mathcal{A}$.

The case of a ring of $S$-integers is slightly more delicate since, as in the case of local rings, neither property $\mathcal{A}$ nor $\mathcal{C}$ holds in general. We will nevertheless prove in section 2 that if $\mathrm{N}$ is hyperbolic at every dyadic place, then for a certain sequence $f$ and for $s$ big enough, properties $\mathcal{C}\left(\mathrm{M} \perp \mathrm{N}^{s}, \mathrm{~N}\right)$ and $\mathcal{A}\left(\mathrm{M} \perp \mathrm{N}^{s}, \mathrm{~N}, f\right)$ hold. In all this section, Kneser's strong approximation theorem will be a crucial tool.

Finally, we prove that in the case of a dyadic local ring (resp. of an $S$-arithmetic ring as in the theorem), the case of hyperbolic N's (resp the case of N's that are hyperbolic at each dyadic place) implies the general case (see subsection 2.6).

As the referee pointed out to the author, the method we use cannot cover the totally real arithmetic case (i.e. all archimedean places are real, and $\Omega$ contains only these real places) except in some particular instances (e.g when $\mathrm{N} \otimes_{\sigma} \mathbb{R}$ is indefinite for at least one imbeding $A \rightarrow \mathbb{R}$ ). An interesting open case is when $A=\mathbb{Z}, \mathrm{M}=\left(\mathbb{Z}, h_{M}\right)$ defined by $h_{M}(x)=-x^{2}$ and $\mathrm{N}=\left(\mathbb{Z}, h_{N}\right)$ defined by $h_{N}(x)=x^{2}$. The theorem would then say that the homology of $\mathrm{O}_{1, n}(\mathbb{Z})$ stabilizes. Let us briefly explain why our method cannot be used to check property $\mathcal{A}$ in that case. We would have to prove that for any $k \geq 1$ there exists an $s$ big enough so that for any choice of subspaces $N_{1}, \ldots, N_{k}$ of $\mathrm{U}_{s}=\mathrm{M} \perp \mathrm{N}^{s}$, each of which is isomorphic to $\mathrm{N}$, the orthogonal Q of $\sum_{i=1}^{k} N_{i}$ contains a copy of $\mathrm{N}$. Our method consists in proving that this holds locally, and then using the strong approximation theorem to deduce that it holds globally. One of the hypotheses of this theorem is that $\mathrm{Q}$ be isotropic at at least one place in $\Omega$ (which in our case is reduced to the real place of $\mathbb{Q}$ ). Now the module $\mathrm{M} \perp \mathrm{N}^{\perp 2}$ can be generated by 3 copies of $\mathrm{N}$, and its orthogonal $\mathrm{Q}$ in $\mathrm{U}_{s}$ is positive definite positive, so we cannot use the strong approximation theorem in this situation. In fact, for any positive definite unimodular $h$-module $\mathrm{T}$ of rank $u$, there exists an isomorphism $\mathrm{M} \perp \mathrm{N}^{\perp 2} \perp \mathrm{T} \simeq \mathrm{M} \perp \mathrm{N}^{\perp u+2}$, and it is well known that for any $u \geq 14$ there exists such T's that do not represent $\mathrm{N}$.

- The particular case $R=\mathbb{Z}\left[\frac{1}{2}\right]$.

Better sequences $f$ than the coarse one given in our theorem can be obtained in a number of particular cases. We will not try to get systematically the sharpest bounds our method can give, except in the following very particular case, which in fact motivated this work. Let $R$ be either a field or a Dedekind domain. We denote by $\mathrm{I}_{n}(R)$ the standard h-module over $R$ (the one whose underlying module is $R^{n}$ and whose bilinear form has the identity matrix as a Gram matrix in the canonical basis) and $\mathrm{O}_{n}(R)$ the group of automorphisms of $\mathrm{I}_{n}(R)$. The properties

$\mathrm{C} 1(R): \mathrm{O}_{n}(R)$ is generated by reflections with respect to hyperplanes,

$\mathrm{C} 2(R, \gamma)$ : for $n \geq \gamma$, the commutator subgroup of $\mathrm{O}_{n}(R)$ is the subgroup $\mathrm{SO}_{n}^{\prime}(R)$ of elements with trivial determinant and spinor norm,

$\mathrm{C} 3(R, \gamma)$ : for $n \geq \gamma, \mathrm{SO}_{n}^{\prime}(R)$ is perfect,

are well known to hold for $\gamma$ big enough in a number of cases (e.g. when $R$ is a finite field with $\gamma=5$ ). When they do, we denote by $\mathrm{BO}_{\infty}^{+}(R)$ the result of Quillen's + construction on $\mathrm{BO}_{\infty}(R)$ relative to the group $\mathrm{SO}_{\infty}^{\prime}(R)$.

In the last section, we shall see these three properties hold with $\gamma=7$ when $R$ is the ring $\mathbb{Z}\left[\frac{1}{2}\right]$. By taking a closer look at this special case, we shall prove the following result.

Theorem (special case). For any integer $k$ and for any integer $n \geq 3 k+5$, the inclusion $\mathrm{O}_{n}\left(\mathbb{Z}\left[\frac{1}{2}\right]\right) \rightarrow$ $\mathrm{O}_{n+1}\left(\mathbb{Z}\left[\frac{1}{2}\right]\right)$ induces an epimorphism (resp. isomorphism) in l-dimensional homology as soon as $l \leq k+1$ (resp. $l \leq k)$. 
Our interest in this case comes from a work of H.-W. Henn and J. Lannes. Indeed, these authors have shown that for $n \leq 14$, the map

$$
\rho_{n}: \mathrm{H}_{*}\left(\mathrm{O}_{n}\left(\mathbb{Z}\left[\frac{1}{2}\right]\right) ; \mathbb{Z}_{(2)}\right) \rightarrow \mathrm{H}_{*}\left(\mathrm{O}_{n}(\mathbb{Z} / 3 \mathbb{Z}) ; \mathbb{Z}_{(2)}\right)
$$

is an isomorphism. We will use this result in order to prove (in the last subsection) the following

Corollary. There are isomorphisms

$$
\pi_{1}\left(\mathrm{BO}_{\infty}^{+}\left(\mathbb{Z}\left[\frac{1}{2}\right]\right)\right) \simeq(\mathbb{Z} / 2)^{2}, \pi_{2}\left(\mathrm{BO}_{\infty}^{+}\left(\mathbb{Z}\left[\frac{1}{2}\right]\right)\right) \simeq \mathbb{Z} / 2, \pi_{3}\left(\mathrm{BO}_{\infty}^{+}\left(\mathbb{Z}\left[\frac{1}{2}\right]\right)\right) \otimes \mathbb{Z}_{(2)} \simeq \mathbb{Z} / 8
$$

In fact, we conjecture that $\rho_{n}$ (which is known to be onto) is an isomorphism in the stable range.

\section{ON THE TOPOLOGY OF AN ADJACENCY RELATION}

\subsection{Pre-simplicial sets and their geometric realization.}

\subsubsection{Pre-simplicial objects.}

For a natural integer $k$, we put $[k]=\{0, \ldots, k\}$ and call $\Delta$ the category whose objects $\operatorname{Ob}(\Delta)$ are the sets $[k]$ and whose morphisms $\operatorname{Mor}(\Delta)$ are the strictly increasing maps. As is well known, such a morphism is a composite of the elementary maps $d_{i}^{k+1}$ defined by

$$
\begin{aligned}
d_{i}^{k+1}:\{0, \ldots, k\} & \rightarrow\{0, \ldots, k+1\} \\
j & \mapsto\left\{\begin{array}{ll}
j & \text { if } j \leq i \\
j+1 & \text { if } j>i
\end{array} .\right.
\end{aligned}
$$

When $\mathcal{H}$ is a category, one says that a contravariant (resp. covariant) functor $\Delta \rightarrow \mathcal{H}$ is a pre-simplicial (resp. pre-cosimplicial) object in $\mathcal{H}$.

Example: Let $\mathrm{E}=(E, \leq)$ be a poset. A flag in $\mathrm{E}$ is a strictly increasing sequence. Let $S_{k}$ be the set of flags of length $k$ in $E$, and let $\partial_{i}^{k+1}$ be the map $S_{k+1} \rightarrow S_{k}$ defined by sending a flag $\left(e_{0}<\cdots<e_{i-1}<e_{i}<\right.$ $\left.e_{i+1}<\cdots<e_{k+1}\right)$ to the flag $\left(e_{0}<\cdots<e_{i-1}<e_{i+1}<\cdots<e_{k+1}\right)$. The functor PS(E) defined on objects by $[k] \rightarrow S_{k}$ and on morphisms by $d_{i}^{k+1} \mapsto \partial_{i}^{k+1}$ is a pre-simplicial set.

The empty pre-simplicial set is written $\phi$.

\subsubsection{Geometric realization.}

Let $E$ be a finite set. The standard simplex on $E$ is the subspace $\underline{\Delta}(E)$ of $\mathbb{R}^{E}$ defined by

$$
\underline{\Delta}(E)=\left\{\sum_{e \in E} x_{e} . e, x_{e} \in[0,1], \sum_{e \in E} x_{e}=1\right\} .
$$

One obtains a pre-cosimplicial topological space $\underline{\Delta}$ by taking for $\underline{\Delta}\left(d_{i}^{k}\right)$ the natural inclusion $\underline{\Delta}([k]) \rightarrow$ $\Delta\left(d_{i}^{k}([k])\right) \rightarrow \Delta[k+1]$.

Let $S: \Delta \rightarrow \mathcal{E} n s$ be a pre-simplicial (discrete) set, and consider on the topological space $\coprod_{k} S_{k} \times \Delta^{[k]}$ the equivalence relation

$$
\left[(x, y) \sim\left(x^{\prime}, y^{\prime}\right)\right] \Leftrightarrow\left[\exists m \in \operatorname{Mor}(\Delta), x=S(m)\left(x^{\prime}\right), y^{\prime}=\underline{\Delta}(m)(y)\right] .
$$

The geometric realization $|S|$ of $S$ is the CW-complex $\bigsqcup_{k} S_{k} \times \Delta^{[k]} / \sim$. A pre-simplicial set $S$ is said to be $n$-acyclic $(n \in \mathbb{Z})$ if its geometric realization is (in particular, $S$ is -1-acyclic iff it is not the empty pre-simplicial set, and all pre-simplicial sets are $n$ acyclic for $n \leq-2$ ). 


\subsubsection{Coverings.}

Let $X: \Delta \rightarrow \mathcal{E} n s$ be a pre-simplicial set. A sub-pre-simplicial set $S$ of $X$ is a pre-simplicial set such that $S_{k}$ is contained in $X_{k}$ for all $k$ 's and such that the restriction $\left.X(m)\right|_{S_{k}}$ and $S(m)$ coincide for any $m \in \operatorname{Mor}_{\Delta}\left([k],\left[k^{\prime}\right]\right)$.

A covering of $X$ is a family $\mathcal{C}=\left(X_{i}\right)_{i \in I}$ of sub-pre-simplicial sets indexed by a totally ordered set $I$, such that for all $n$ the equality $\cup_{I} X_{i}([n])=X([n])$ holds. The nerve of such a covering is the geometric realization $\mathrm{N}(\mathcal{C})=|N(\mathcal{C})|$ where $N(\mathcal{C})$ is the pre-simplicial set defined by

$$
N(\mathcal{C})([n])=\left\{\text { subfamilies }\left(i_{0}<\cdots<i_{n}\right) \text { of } I \text { such that } \cap_{k=0}^{n} X_{i_{k}} \neq \phi\right\}
$$

with the obvious face maps.

The following result is not surprising (a proof is obtained by mimicking [Brown, VII-4-4]).

Proposition 1. Let $n$ be an integer, $X$ be a pre-simplicial set and $\mathcal{C}=\left(X_{i}\right)_{i \in I}$ be a covering of $X$.

If for all non-empty finite $J \subset I$, the sub-pre-simplicial set $\cap_{j \in J} X_{j}$ is $n-\operatorname{card}(J)+1$-acyclic, then the pre-simplicial set $X$ is $n$-acyclic.

\subsection{The criterion.}

\subsubsection{Adjasets.}

Let $E$ be a set. An adjacency relation on $E$ is a symmetric relation $\perp$ such that $x \perp y$ implies $x \neq y$. For convenience, we introduce the neologism adjaset for a datum $\mathrm{E}=(E, \perp)$. ) .

Let $\mathrm{E}=(E, \perp)$ be an adjaset and let $P \subset E$ be a subset. We write $\operatorname{Ad}_{\mathrm{E}}(P)=(\{x \in E, \forall p \in P, x \perp p\}, \perp$

\subsubsection{Geometric realizations.}

To an adjaset $\mathrm{E}$ we associate the sets $\mathrm{S}(\mathrm{E})[k]$ of maps $x:[k] \rightarrow E$ satisfying the property

$$
\forall s, t \in[k], \quad s \neq t \Rightarrow x(s) \perp x(t)
$$

and the union $S(\mathrm{E})$ of all $\mathrm{S}(\mathrm{E})[k]$ 's.

On one hand, we may turn $k \mapsto \mathrm{S}(\mathrm{E})[k]$ into a pre-simplicial set (which we denote by $\mathrm{S}(\mathrm{E})$ ) by associating to an element $m \in \operatorname{Mor}_{\Delta}\left(\left[k^{\prime}\right],[k]\right)$ the map $\mathrm{S}(\mathrm{E})(m)$ defined by $\mathrm{S}(\mathrm{E})(m)(x)=x \circ m$. We put $|\mathrm{E}|=|\mathrm{S}(\mathrm{E})|$.

On the other hand, we may define on $S(\mathrm{E})$ the following order relation:

$$
x \leq y \Leftrightarrow \exists m \in \operatorname{Mor}(\Delta), x=y \circ m .
$$

This makes of $(S(\mathrm{E}), \leq)$ a poset, the geometric realization $|\mathrm{PS}(S(\mathrm{E}), \leq)|$ of which we write $\|\mathrm{E}\|$.

We note that $\|\mathrm{E}\|$ is nothing but the barycentric subdivision of $|E|$, so that these two topological spaces are homotopy equivalent.

\subsubsection{Strong acyclicity.}

We say that a non-empty adjaset $\mathrm{E}$ is strongly $n$-acyclic if the adjaset $\operatorname{Ad}_{\mathrm{E}}(P)$ is $(n-\operatorname{card}(P)+1)$-acyclic for all subsets $P \subset E$ satisfying $\operatorname{card}(P) \geq 2$, and extend this definition by saying that a non-empty adjaset is -1 -acyclic.

Proposition 2. Let $\mathrm{E}=(E, \perp)$ be a strongly n-acyclic adjaset.

$\circ \mathrm{E}$ is n-acyclic ;

- $\operatorname{Ad}_{E}(x)$ is $(n-1)$-acyclic for all $x \in \mathrm{E}$. 
Proof:

- Let us begin with some generalities. For two posets $\mathrm{X}_{1}=\left(X_{1}, \leq_{1}\right)$ and $\mathrm{X}_{2}=\left(X_{2}, \leq_{2}\right)$, the Hopf construction $^{2} H\left(X_{1}, X_{2}\right)$ is the poset whose underlying set is the disjoint union $X_{1} \bigsqcup X_{2} \bigsqcup X_{1} \times X_{2}$ and whose order is defined by

$$
x \leq y \text { if and only if }
$$

$\begin{array}{llcl}\text { either } & x \in X_{1}, & y \in X_{1} & \text { and } x \leq_{1} y \\ \text { or } & x \in X_{2}, & y \in X_{2} & \text { and } x \leq_{2} y \\ \text { or } & x \in X_{1}, & y=(s, t) \in X_{1} \times X_{2} & \text { and } x \leq_{1} s \\ \text { or } & x \in X_{2}, & y=(s, t) \in X_{1} \times X_{2} & \text { and } x \leq_{2} t \\ \text { or } & x=(s, t) \in X_{1} \times X_{2}, & y=(u, v) \in X_{1} \times X_{2} & \text { and } s \leq_{1} u, t \leq_{2} v .\end{array}$

The geometric realization $\left|\mathrm{PS}\left(H\left(\mathrm{X}_{1}, \mathrm{X}_{2}\right)\right)\right|$ is then homeomorphic to the topological join $\left|\mathrm{PS}\left(\mathrm{X}_{1}\right)\right| \star\left|\mathrm{PS}\left(\mathrm{X}_{2}\right)\right|$. By the way, recall that if $T_{1}$ is a $k_{1}$-acyclic $\mathrm{CW}$-complex, and if $T_{2}$ is a $k_{2}$-acyclic $\mathrm{CW}$-complex, then the join $T_{1} \star T_{2}$ is $\left(k_{1}+k_{2}+2\right)$-acyclic.

○ We associate to $x \in E$ the sub-poset $U_{x}$ of $(S(\mathrm{E}), \leq)$ defined by

$$
U_{x}=S\left(\operatorname{Ad}_{E}(x)\right) \cup\{s \in S(\mathrm{E}), s(0)=x\},
$$

and to a subset $P \subset E$ the sub-poset

$$
U_{P}:=\cap_{x \in P} U_{x} .
$$

Then the family of pre-simplicial sets $\left(\operatorname{PS}\left(U_{x}, \leq\right)\right)_{x \in E}$ is a covering of $\operatorname{PS}(S(\mathrm{E}), \leq)$. Given a subset $P \subset E$, let us call $P_{0}$ the (discrete) poset $\left\{x \in P, x \in \operatorname{Ad}_{(P, \perp)}(P-x)\right\}$; then the natural map

$$
H\left(P_{0}, S\left(\operatorname{Ad}_{E}(P)\right)\right) \rightarrow U_{P}
$$

is an isomorphism of posets.

- In order to deduce the first point from proposition 1 , it is enough to show that for any subset $P \subset E$, the space $\left\|U_{P}\right\|$ is $n-\operatorname{card}(P)+1$-acyclic. If $\operatorname{card}(P)=1$, then the homeomorphism $\left\|U_{x}\right\| \simeq\|x\| \star\left\|\operatorname{Ad}_{\mathrm{E}}(x)\right\| \operatorname{implies}$ that $\left\|U_{x}\right\|$ is contractible. If $\operatorname{card}(P) \geq 2,\left\|U_{P}\right\|$ is homeomorphic to the join of $\left\|P_{0}\right\|$ and $\left\|\operatorname{Ad}_{\mathrm{E}}(P)\right\|$. Since by hypothesis, $\left\|\operatorname{Ad}_{\mathrm{E}}(P)\right\|$ is $(n-\operatorname{card}(P)+1)$-acyclic, so is $\left\|U_{P}\right\|$.

$\circ$ A similar argument proves the second point.

In particular, for $\mathrm{E}$ to be strongly $n$-acyclic, it is enough that $\operatorname{Ad}_{\mathrm{E}}(P)$ be $(n-\operatorname{card}(P)+1)$-strongly acyclic for any subset $P$ such that $\operatorname{card}(P) \leq n+2$. From this remark, one immediately deduces the

Acyclicity criterion: Let $\mathrm{E}=(E, \perp)$ be an adjaset and $k$ an integer. If for any family $\left(P_{1}, \ldots, P_{u}\right)$ of finite subsets of E such that

$$
\operatorname{card}\left(P_{i}\right) \geq 2, i \neq j \Rightarrow P_{i} \perp P_{j} \text { and } \sum_{i}\left(\operatorname{card}\left(P_{i}\right)-1\right) \leq k+1
$$

the set $\left(\cup_{i} P_{i}\right)^{\perp}$ is not empty, then $\mathrm{E}$ is k-acyclic.

\section{On properties $\mathcal{C}$ and $\mathcal{A}$ for hermitian modules}

\subsection{Hermitian modules over composition algebras.}

\footnotetext{
${ }^{2}$ According to the referee, this construction has been introduced and studied by Hendrik Maazen in his thesis, and so christened to avoid confusion with the classical join construction for posets.
} 
2.1.1. Composition algebras over fields.

If $R$ is a (commutative) field, a composition algebra $\mathrm{C}=(C, N)$ over $R$ is the data of a finite dimensional $R$-algebra $C$ with unit $e$ together with a non-degenerate quadratic form $N: C \rightarrow R$ such that

$$
N(x y)=N(x) N(y) \quad(\forall x, y \in C) .
$$

Here, non-degenerate means that the associated bilinear form

$$
b(x, y)=N(x+y)-N(x)-N(y)
$$

induces an isomorphism

$$
\begin{aligned}
\psi: C & \rightarrow \operatorname{Hom}_{R}(C, R) \\
x & \mapsto[y \mapsto b(x, y)]
\end{aligned}
$$

Such a composition algebra comes with a natural anti-involution (called the conjugation in $\mathrm{C}$ ) $x \mapsto \bar{x}=$ $b(x, e) e-x$. Put $\operatorname{Tr}(x)=x+\bar{x}$. Then one has the properties

$\circ x \bar{x}=\bar{x} x=N(x) e$,

$\circ x=\bar{x} \Leftrightarrow x \in R . e$,

○ the polynomial $\chi_{x}(Z)=Z^{2}-\operatorname{Tr}(x) Z+N(x) e$ is characteristic for $x\left(\right.$ i.e. $\left.\chi_{x}(x)=0\right)$.

Here are the possibilities for composition algebras over fields:

(D1): $C$ is 1-dimensional over $R, C=R, N(x)=x^{2}$, and $\bar{x}=x$,

(D2S): $C$ is 2-dimensional split over $R, C=R \times R, N((\alpha, \beta))=\alpha \beta$, and $\overline{(\alpha, \beta)}=(\beta, \alpha)$,

(D2NS): $C$ is 2-dimensional non-split over $R, C=R[X] / X^{2}-\theta$ for some non-square $\theta \in R, N(\alpha+\beta X)=$ $\alpha^{2}-\theta \beta^{2}$, and $\overline{\alpha+\beta X}=\alpha-\beta X$,

(D4S): $C$ is 4-dimensional split over $R, C=\mathrm{M}_{2}(R), N(x)=\operatorname{det}(x)$ and $\left[\begin{array}{ll}\alpha & \beta \\ \gamma & \delta\end{array}\right]=\left[\begin{array}{cc}\delta & -\beta \\ -\gamma & \alpha\end{array}\right]$,

(D4NS): $C$ is 4-dimensional non-split over $R$, and is an anisotropic quaternion algebra $\mathrm{C}=(C, N)=\left(\frac{u, v}{K}\right)$ defined by

$$
\begin{gathered}
C e=R+R i+R j+R i j \text { with } i^{2}=-u e, j^{2}=-v e, i j=-j i \\
\alpha e+\beta i+\gamma j+\delta k=\alpha e-\beta i-\gamma j-\delta k \text { and } N(a) e=a \cdot \bar{a} .
\end{gathered}
$$

(D8): $C$ is 8 -dimensional and non-associative.

In what follows, we will always assume our composition algebras to be associative.

\subsubsection{Composition algebras over local rings and rings of $(S)$-integers.}

Let $K$ be a number field with ring of integers $\mathcal{O}_{K}, \mathcal{V}$ (resp. $\mathcal{V}^{f}$, resp. $\mathcal{V}^{\infty}$ ) its set of places (resp. non-archimedean places, resp. archimedean places), $\Omega$ a finite subset of $\mathcal{V}$ containing $\mathcal{V}^{\infty}$, and $S=\mathcal{V}-\Omega$.

For $\mathfrak{p}$ in $\mathcal{V}$, let $K_{\mathfrak{p}}$ be the completion of $K$ at $\mathfrak{p}$. If $\mathfrak{p}$ is non-archimedean (resp. archimedean), let $\mathcal{O}_{K_{\mathfrak{p}}}$ be the ring of integers of $K_{\mathfrak{p}}$ (resp. put $\mathcal{O}_{K_{\mathfrak{p}}}=K_{\mathfrak{p}}$ ). We also use the notation $\mathfrak{p}$ for the maximal ideal in $\mathcal{O}_{K_{\mathfrak{p}}}$.

The ring of $(S)$-integers of $K$ is the subset $\mathcal{O}_{K, S}$ of elements that are in $\mathcal{O}_{K_{\mathfrak{p}}}$ for all $\mathfrak{p} \in S$.

In this paper, we define a composition algebra $\mathrm{C}=(C, N)$ over $\mathcal{O}_{K_{\mathfrak{p}}}$ to be a maximal $\mathcal{O}_{K_{\mathfrak{p}}}$-order $C$ in a composition algebra $\left(C_{K_{\mathfrak{p}}}, N\right)$ over $K_{\mathfrak{p}}$. Note that the form of such a maximal order is quite restricted:

○ if $C_{K_{\mathfrak{p}}}$ is 1-dimensional, then $C=\mathcal{O}_{K_{\mathfrak{p}}}$.

○ if $C_{K_{\mathfrak{p}}}$ is 2-dimensional non split, then it is a (commutative) field and $C=\mathcal{O}_{C_{K_{\mathfrak{p}}}}$ is its ring of integers, which is a local ring. If $\mathfrak{p} C$ is the maximal ideal of $C$, then we say $\mathrm{C}$ is of type D2NSI (I stands for inert). If $\mathfrak{p} C$ is not the maximal ideal $\mathfrak{q}$ of $C$, then we have $\mathfrak{q}^{2}=\mathfrak{p} C$, and we say $\mathrm{C}$ is of type D2NSR (R stands for ramified).

$\circ$ if $C_{K_{\mathfrak{p}}}=K_{\mathfrak{p}} \times K_{\mathfrak{p}}$ is 2-dimensional split, then $C_{\mathfrak{p}}=\mathcal{O}_{K_{\mathfrak{p}}} \times \mathcal{O}_{K_{\mathfrak{p}}}$.

$\circ$ if $C_{K_{\mathfrak{p}}}=\mathrm{M}_{2}\left(K_{\mathfrak{p}}\right)$ is 4-dimensional split, then $C_{\mathfrak{p}} \simeq \mathrm{M}_{2}\left(\mathcal{O}_{K_{\mathfrak{p}}}\right)$. 
○ if $C_{K_{\mathfrak{p}}}$ is 4-dimensional non split, then $C_{K_{\mathfrak{p}}}=\left(\frac{\pi, \Delta}{K_{\mathfrak{p}}}\right)$, where $\pi$ is a uniformiser for $\mathfrak{p}$ and $\Delta$ is a unit of maximal quadratic defect (see [O'Meara 63:17]), is a skew field and $C_{\mathfrak{p}}=\mathcal{O}_{C_{K_{\mathfrak{p}}}}$ is its ring of integers (i.e. the subset of elements $x \in C_{K_{\mathfrak{p}}}$ whose characteristic polynomial $\chi_{x}$ have coefficients in $\mathcal{O}_{K_{\mathfrak{p}}}$.

Finally, we define a composition algebra $\mathrm{C}=(C, N)$ over $\mathcal{O}_{K, S}$ to be a maximal $\mathcal{O}_{K, S}$-order $C$ in a composition algebra $\left(C_{K}, N\right)$ over $K$. As is well known, this is equivalent to requiring that $C_{\mathfrak{p}}:=\mathcal{O}_{K_{\mathfrak{p}}} \otimes_{\mathcal{O}_{K, S}} C$ be a maximal order in $\left(C_{K}\right)_{\mathfrak{p}}$ at each place $\mathfrak{p} \in S$.

\subsubsection{Hermitian modules over composition algebras.}

Let $R$ be a commutative ring with unit, and $C$ an associative $R$-algebra together with an anti-automorphism $c \mapsto \bar{c}$ centralizing $R$.

Let $M$ be a left $C$-module. The dual module $\operatorname{Hom}_{C}(M, C)$ is made into a left $C$-module by putting

$$
(\lambda . \varphi)(x)=\varphi(x) \bar{\lambda} .
$$

A hermitian module is the datum $\mathrm{M}=(M, h)$ of a projective left $C$-module $M$ of finite type, together with a hermitian form relative to $C$, i.e. a function $h: M \times M \rightarrow C$ such that $h(x, y)=\overline{h(y, x)}$ and $h(a x, b y)=a h(x, y) \bar{b}$. In particular, one has $h(x, x) \in R$ and $h(\lambda x, \lambda x)=N(\lambda) h(x, x)$ for all $x \in M$ and $\lambda \in C$.

When $k$ is a (commutative) $R$-algebra, we write $\mathrm{M}_{k}=\left(M_{k}, h_{k}\right)$ for the left $\mathrm{C}_{k}$-module $M_{k}=k \otimes_{R} M$ together with the hermitian form $h_{k}$ obtained from $h$ by extension of scalars.

We will say that the hermitian module $\mathrm{M}$ is an h-module (resp. an $\tilde{\mathrm{h}}$-module) if the associated morphism of left $C$-modules

is an isomorphism (resp. is injective).

$$
\begin{aligned}
\Psi_{h}: M & \rightarrow \operatorname{Hom}_{C}(M, C) \\
y & \mapsto[x \mapsto h(x, y)]
\end{aligned}
$$

Of course, when $R$ is a field, both notions coincide. Moreover, we have

Lemma 3. Let $R$ be a field, let $\mathrm{C}=(C, N)$ be a composition algebra over $R$ and let $\mathrm{M}=(M, h)$ be an $\mathrm{h}$-module over $\mathrm{C}$. Then $M$ is a free $C$-module, and if $\mathrm{C}$ is split, $\mathrm{M}$ admits an orthonormal basis. Moreover, a $C$-submodule of $M$ that can be generated by $m$ vectors is contained in an $\mathrm{h}$-submodule of $\mathrm{M}$ of rank no larger than $2 m$.

Proof of the first point: Assume $\mathrm{C}$ is of type D2S. Write $C=R \times R$, put $\varepsilon_{1}=(1,0), \varepsilon_{2}=(0,1)$, and consider $M_{1}=\varepsilon_{1} M, M_{2}=\varepsilon_{2} M$. Then $M_{1}$ and $M_{2}$ are totally isotropic (i.e. $h(x, y)=0$ for $x, y \in M_{i}$ ), and $M$ decomposes as a direct sum $M_{1} \oplus M_{2}$. Let $x$ be an element of $M_{1}$. There exists an element $y$ in $M_{2}$ such that $h(x, y)=\varepsilon_{1}$. Thus $h(x+y, x+y)=e$. The submodule $C .(x+y)$ is free, and splits. An induction argument furnishes an orthonormal basis for $M$.

Assume $\mathrm{C}$ is of type $\mathrm{D} 4 \mathrm{~S}$. Write $C=\mathrm{M}_{2}(R)$ is of type $\mathrm{D} 4 \mathrm{~S}$, put $\varepsilon_{1}=\left[\begin{array}{ll}1 & 0 \\ 0 & 0\end{array}\right] \in C$ and $\varepsilon_{2}=\left[\begin{array}{ll}0 & 0 \\ 0 & 1\end{array}\right] \in C$. Let $x$ be a non-trivial vector of $\varepsilon_{1} M$. Then $h(x, x)$ is null, since it has to be a scalar multiple of $e=\left[\begin{array}{ll}1 & 0 \\ 0 & 1\end{array}\right]$ that lies in $\left[\begin{array}{ll}0 & * \\ 0 & 0\end{array}\right]$. Now the restriction $h: \varepsilon_{1} M \times \varepsilon_{2} M \rightarrow R$ is $R$-bilinear, with values in $\left[\begin{array}{cc}* & 0 \\ 0 & 0\end{array}\right]$ and is non-degenerate. Thus one can find a vector $y$ in $\varepsilon_{2} M$ such that $h(x, y)=\varepsilon_{1}$, and one has $h(x+y, x+y)=e$. An induction argument furnishes an orthonormal basis for $M$. The second point admits a similar proof.

When $R$ is either a local ring or a ring of $(S)$-integers with field of fractions $K$, and $\mathrm{C}=(C, N)$ is a composition algebra over $R$, we define the rank of $\mathrm{M}$ to be the dimension over $C_{K}$ of the free module $M_{K}$. We associate to $M$ its dual $M^{\sharp}$ which is defined by

$$
M^{\sharp}=\left\{x \in M_{K}, h(x, m) \in \mathrm{C} \forall m \in M\right\} .
$$

$\circ M^{\sharp}$ is a C-module that contains $M$, 
$\circ \mathrm{M}$ is an $\tilde{\mathrm{h}}$-module iff $\mathrm{M}_{K}$ is an h-module, and then $M^{\sharp}=\left(\Psi_{h_{K}}\right)^{-1}\left[\operatorname{Hom}_{C}(M, C)\right]$ is a C-lattice in $M_{K}$, $\circ \mathrm{M}$ is an h-module iff $M^{\sharp}=M$.

Examples: Let $R$ be either a field $K$, a local ring with field of fractions $K$, or an $(S)$-arithmetic ring with field of fractions $K$ and let $\mathrm{C}=(C, N)$ be a composition algebra over $R$.

○ For any element $r \in R$, the hermitian module $\left\langle r>_{C}=(C, h)\right.$ defined by $h(x, y)=r x \bar{y}$ is an h-module when $r$ is invertible in $R$ and an $\tilde{\mathrm{h}}$-module when $r$ is non-zero.

- For any element $\varepsilon \in R^{\times}$, the h-module $\langle 1\rangle^{\perp n-1} \perp\langle\varepsilon\rangle$ will be denoted by $\mathrm{I}_{n}^{(\varepsilon)}(\mathrm{C})$. For $\varepsilon=1$, we denote it by $\mathrm{I}_{n}(\mathrm{C})$.

- Let $c$ be an element of $C$. The hermitian module $\mathrm{H}^{(c)}(\mathrm{C})=(C \times C, h)$ defined by $h\left(\left(x, x^{\prime}\right),\left(y, y^{\prime}\right)\right)=$ $x c \bar{y}^{\prime}+x^{\prime} \bar{c} \bar{y}$ is an $\tilde{\mathrm{h}}$-module when $c$ becomes invertible in $K \otimes_{R} C$ and is an h-module when $c$ is invertible in $C$. For $c=1$, we denote it by $\mathrm{H}(\mathrm{C})$ and call it the hyperbolic plane. An h-module over $\mathrm{C}$ is said to be hyperbolic if it decomposes as an orthogonal sum of hyperbolic planes.

- Let $\mathrm{M}=(M, h)$ be a hermitian module over $\mathrm{C}$, and $r$ an element of $R$. We write $\langle r\rangle \otimes \mathrm{M}$ for the hermitian module $\left(M, h^{\prime}\right)$ defined by $h^{\prime}(x, y)=r \cdot h(m, n)$. More generally, if $\mathrm{N}$ is a hermitian module over $R$, we denote by $\mathrm{N} \otimes \mathrm{M}$ the hermitian module $\left(N \otimes M, h_{N} \otimes h_{M}\right)$ defined by $h_{N} \otimes h_{M}\left(n \otimes m, n^{\prime} \otimes m^{\prime}\right)=h_{N}\left(n, n^{\prime}\right) h_{M}\left(m, m^{\prime}\right)$.

- When the context makes it superfluous, we omit mention of $\mathrm{C}$ and write $\mathrm{I}_{n}^{\varepsilon}$ and $\mathrm{I}_{n}, \mathrm{H}$ and so on.

Let $\mathrm{C}$ be a composition algebra over $R$ and let $\mathrm{M}=(M, h)$ be a hermitian module over $\mathrm{C}$. The elements of $\operatorname{Hom}_{C}(M, M)$ act on the right on $M$ (so that $(\lambda x) \cdot g=\lambda(x . g)$ ). The group of invertible elements $g$ in $\operatorname{Hom}_{C}(M, M)$ such that $h(x . g, y . g)=h(x, y)$ for all $x, y \in M$ will be written $\mathrm{U}(\mathrm{M})$. The following remark is classical.

Remark: if $\mathrm{C}$ is of type $\mathrm{D} 2 \mathrm{~S}$, and $\mathrm{M}$ is of rank $n$, then the projection $\mathrm{U}(\mathrm{M}) \rightarrow \operatorname{Aut}_{R}\left(\varepsilon_{1} M\right) \simeq \mathrm{GL}_{n}(R)$ is an isomorphism, where $\varepsilon_{1}=(1,0)$ as above.

If $\mathrm{C}$ is of type $\mathrm{D} 4 \mathrm{~S}$ and $\mathrm{M}$ is of rank $n$, then on the $R$-vector space $\varepsilon_{1} M$, where $\varepsilon_{1}=\left[\begin{array}{ll}1 & 0 \\ 0 & 0\end{array}\right]$ as above, $h$ is $R$-bilinear and alternating with values in $\left[\begin{array}{ll}0 & * \\ 0 & 0\end{array}\right]$ and the projection $\mathrm{U}(\mathrm{M}) \rightarrow \operatorname{Aut}\left(\varepsilon_{1} M, h\right) \simeq \operatorname{Sp}_{2 n}^{s}(R)$ furnishes an isomorphism between the unitary group of $\mathrm{M}$ and the split form over $R$ of the symplectic group.

\subsubsection{Properties $\mathcal{C}$ and $\mathcal{A}$.}

Let $R$ be either a field, a local ring or an $(S)$-arithmetic ring, and let $\mathrm{C}$ be a composition algebra over $R$. Recall from the introduction that we are interested in the following properties, defined for two h-modules $\mathrm{M}$ and $\mathrm{N}$ over $\mathrm{C}$, and a sequence $f=\left(f_{k}\right)_{k \geq 0}$ of integers such that $f_{k} \geq 2 k+1$.

Property $\mathcal{C}(\mathrm{M}, \mathrm{N})$ : let $\mathrm{B}$ be an h-module over $\mathrm{C}$. If there exists an isomorphism $\mathrm{B} \perp \mathrm{N} \simeq \mathrm{M} \perp \mathrm{N}^{k+1}$ for some integer $k$, then there exists an isomorphism $\mathrm{B} \simeq \mathrm{M} \perp \mathrm{N}^{k}$.

Property $\mathcal{A}(\mathrm{M}, \mathrm{N}, f)$ : for all $n \geq f_{k}$, for all $k$-test sequences $\left(P_{1}, \ldots, P_{s}\right)$ of elements of $\operatorname{Rep}\left(\mathrm{N}, \mathrm{M} \perp \mathrm{N}^{n+1}\right)$ there exist an element $T$ in $\operatorname{Rep}\left(\mathrm{N}, \mathrm{M} \perp \mathrm{N}^{n+1}\right)$ such that $\mathrm{T}$ is orthogonal to all elements of each $P_{i}$.

Let $\mu$ and $\nu$ be respectively the ranks of $\mathrm{M}$ and $\mathrm{N}$. Let

$$
\left\{N_{1}^{1}, \ldots, N_{u_{1}}^{1}\right\} \perp\left\{N_{1}^{2}, \ldots, N_{u_{2}}^{2}\right\} \perp \cdots \perp\left\{N_{1}^{s}, \ldots, N_{u_{s}}^{s}\right\}
$$

be such a $k$-test sequence in $\mathrm{M} \perp \mathrm{N}^{\perp n+1}$; then $P=\sum_{i, j} N_{i}^{j}$ is contained in an $\tilde{\mathrm{h}}$-module $\mathrm{Q}$ of rank no larger than $3(k+1) \nu$ that contains at least $s \geq 1$ direct summands isomorphic to $\mathrm{N}$. We just have to ensure that $\mathrm{N}$ is represented by $\mathrm{Q}^{\perp}$, which is an $\tilde{\mathrm{h}}$-module of rank at least $\mu+(n-3 k-2) \nu$. In all situations we shall encounter, the sequence $\left(f_{k}\right)_{k}$ will be linear in $k$. Moreover we will always work in the presence of property $\mathcal{C}(\mathrm{M}, \mathrm{N})$. Thus we introduce the following property, for $\alpha \geq 2$ and $\beta \geq 1$ (that, in presence of $\mathcal{C}(\mathrm{M}, \mathrm{N})$, implies $\mathcal{A}\left(\mathrm{M}, \mathrm{N}, f_{k}\right)$ with $\left.f_{k}=\alpha k+\beta\right)$ : 
Property $\mathcal{A}(\mathrm{M}, \mathrm{N}, \alpha, \beta)$ : If $n \geq \alpha k+\beta$, the orthogonal complement of any sub-h-hodule of $\mathrm{M} \perp \mathrm{N}^{n}$ of rank no larger than $(3 k+2) \nu$ represents $\mathrm{N}$.

In many cases, property $\mathcal{A}(\mathrm{M}, \mathrm{N}, \alpha, \beta)$ (resp. $\mathcal{C}(\mathrm{M}, \mathrm{N}))$ holds for all modules $\mathrm{M}$ and $\mathrm{N}$ over a given $\mathrm{C}$. In that case, we say that property $\mathcal{A}(\alpha, \beta)$ (resp. $\mathcal{C})$ holds over $\mathrm{C}$.

\subsection{Properties $\mathcal{C}$ and $\mathcal{A}$ when $R$ is a field.}

\subsection{1. $t$-universal composition algebras.}

Note that when $R$ is a field where 2 is invertible, and $\mathrm{C}$ is a composition algebra over $R$ that is non-split, property $\mathcal{C}$ holds by Witt's theorem. It also follows from lemma 3 that property $\mathcal{C}$ holds over a split algebra $\mathrm{C}$ over any field.

A composition algebra $\mathrm{C}$ over a field $R$ will be said to be $t$-universal if any h-module over $\mathrm{C}$ admits an orthogonal basis, and if any h-module over $\mathrm{C}$ of rank $t$ represents all h-modules of rank 1 .

For example,

$\circ \mathbb{C} / \mathbb{C}$ is 1 -universal.

$\circ$ a split composition algebra over a field is 1-universal by lemma 3 .

$\circ$ if $R$ is a finite field of odd characteristic, then $R / R$ is 2 -universal, and if $\mathrm{C} / R$ is of type $\mathrm{D} 2 \mathrm{NS}, \mathrm{C}$ is 1-universal.

$\circ$ If $R$ is a complete local field, then $\mathrm{C} / R$ is 4-universal (resp. 2-universal, resp. 1-universal) if $\mathrm{C}$ is of type D1 (resp. D2NS, resp. D4NS).

Now the following remark is obvious:

Proposition 4. If $C$ is t-universal, property $\mathcal{A}\left(M, N, 3,3+\frac{t-1-\mu}{\nu}\right)$ holds, and in particular, $\mathcal{A}(3, t+2)$ holds over $\mathcal{C}$.

Thus in all the examples listed above, properties $\mathcal{C}$ and $\mathcal{A}$ hold.

\subsubsection{C of type D1 or D2NS over a finite field of characteristic 2.}

Let $\mathrm{M}$ be an h-module over $\mathrm{C}$. Then if $u$ is a non-trivial isotropic vector of $\mathrm{M}$, there exists a vector $y \in M$ such that $h(x, y)$ equal 1 . If $y$ is isotropic, then the submodule $\langle x, y\rangle$ is hyperbolic. If $y$ is non-isotropic, then the submodule $\langle y\rangle$ is an h-submodule. Since the map $C^{\times} \stackrel{N}{\longrightarrow} R^{\times}$is onto, it follows that there exists an isomorphism

$$
\mathrm{N} \simeq \mathrm{H}^{s} \perp\langle 1\rangle^{t}
$$

Now if $\mathrm{C} / R$ is an extension $\mathbb{F}_{q^{2}} / \mathbb{F}_{q}$, then there exists $\varepsilon \in \mathbb{F}_{q}$ such that $\mathbb{F}_{q^{2}}=\mathbb{F}_{q}[X] /\left(X^{2}+X+\varepsilon\right)$. The trace of an element $a+b X$ is then $b$. In particular, $X+\bar{X}=1$. This furnishes an isomorphism

$$
\mathrm{N} \simeq\langle 1\rangle^{2 s+t}
$$

and $\mathrm{C}$ is 1-universal. In particular, properties $\mathcal{C}$ and $\mathcal{A}(3,3)$ hold over $\mathrm{C}$.

If $C=R$, then there exists an isomorphism $\langle 1\rangle^{\perp 3} \simeq \mathrm{H} \perp\langle 1\rangle$, already defined over $\mathbb{F}_{2}$. Thus for any h-module $\mathrm{N}$, there exists a decomposition

$$
\mathrm{N} \simeq \mathrm{H}^{s} \perp\langle 1\rangle^{t}
$$

for a unique integer $t \in\{0,1,2\}$ (note that since any vector of the hyperbolic plane over $R$ is isotropic, there exists no isomorphism $\left.\langle 1\rangle^{\perp 2} \rightarrow \mathrm{H}\right)$. It follows that

o property $\mathcal{C}(\mathrm{M}, \mathrm{N})$ holds for any $\mathrm{M}$ when $\mathrm{N}$ is hyperbolic, $\circ \mathrm{N}^{\perp 3}$ is isomorphic to $\mathrm{N}^{\prime} \perp \mathrm{N}$ with $\mathrm{N}^{\prime}$ hyperbolic for any $\mathrm{N}$,

○ property $\mathcal{A}(\mathrm{M}, \mathrm{N}, 3,4)$ holds for any $\mathrm{M}$ when $\mathrm{N}$ is hyperbolic. 


\subsection{3. $R=\mathbb{R}$ and $\mathrm{C}$ non-split.}

In this situation, there exists for any h-module $\mathrm{M}$ an isomorphism

$$
\mathrm{M} \simeq\langle 1\rangle^{\perp s} \perp\langle-1\rangle^{\perp t} .
$$

The integers $s$ and $t$ are uniquely determined, and the couple $(s, t)$ is called the signature of M.

Proposition 5. If $R=\mathbb{R}$ and $\mathrm{C} / R$ is non-split, then for all $\mathrm{h}$-modules $\mathrm{M}$ and $\mathrm{N}$ of respective ranks $\mu$ and $\nu$ over $\mathrm{C}, \mathcal{A}(M, N, \nu+2, \nu+2)$ holds. If moreover $\mathrm{M}$ and $\mathrm{N}$ are both positive (or both negative) definite, $\mathcal{A}(\mathrm{M}, \mathrm{N}, f)$ holds, with $f_{k}=2 k+2$.

Proof: Let $\left(\mu^{+}, \mu^{-}\right)$be the signature of $\mathrm{M}$ and let $\left(\nu^{+}, \nu^{-}\right)$be the signature of $\mathrm{N}$. The signature of $\mathrm{Q}^{\perp}$ is at worst $\left(\mu^{+}+(n-2 k-1) \nu^{+}-(k+1) \nu, \mu^{-}+(n-2 k-1) \nu^{-}-(k+1) \nu\right)$. Thus $\mathrm{N}$ will be represented by $\mathrm{Q}^{\perp}$ as soon as $n \geq\left(\max \left(\frac{\nu}{\nu^{+}}, \frac{\nu}{\nu^{-}}\right)+2\right) k+\left(\max \left(\frac{\nu}{\nu^{+}}, \frac{\nu}{\nu^{-}}\right)+2\right)$.

\subsection{4. $R$ a global field.}

The following result, known as the Hasse principle, is obvious when $\mathrm{C}$ is split, and follows immediately from the well-known Hasse-Minkowski theorem [O'Meara 66:3] in the other cases.

Theorem 6. Let $R$ be a global field, $\mathrm{C}$ a composition algebra over $R, \mathrm{M}$ and $\mathrm{N}$ two h-modules over $\mathrm{C}$. Then $\mathrm{M}$ represents $\mathrm{N}$ iff $\mathrm{M}_{\mathfrak{p}}$ represents $\mathrm{N}_{\mathfrak{p}}$ at each place $\mathfrak{p}$ of $R$.

Corollary 7. Let $R$ be a global field, $\mathrm{C}$ a composition algebra over $R, \mathrm{M}$ and $\mathrm{N}$ two h-modules over $\mathrm{C}$ of respective ranks $\mu$ and $\nu$. Then property $\mathcal{A}(\mathrm{M}, \mathrm{N}, \nu+2, \max (\nu+2,6))$ holds. If there exists a real place at which $\mathrm{M}$ and $\mathrm{N}$ are both positive (or both negative) definite, then property $\mathcal{A}(\mathrm{M}, \mathrm{N}, f)$ holds with $f_{k}=2 k+2+3 / \nu$.

\subsection{The residue of an $\tilde{h}$-module.}

Let $R$ be either a local ring or a ring of $(S)$-integers, with fraction field $K$. Let $\mathrm{C}=(C, N)$ be a composition algebra over $R$. We have seen that the dual $M^{\sharp}$ of an $\tilde{\mathrm{h}}$-module $\mathrm{M}=(M, h)$ is a $C$-lattice in $\mathrm{M}_{K}=\left(M_{K}, h\right)$ that contains $M$. The quotient $M^{\sharp} / M$ is a left $C$-torsion module and inherits a form, that we still write $h$, defined by

$$
h(x+M, y+M)=h(x, y)+C \in C_{K} / C .
$$

The pair $\left(M^{\sharp} / M, h\right)$ is called the residue of $\mathrm{M}$ and is denoted by $\operatorname{Res}(\mathrm{M})$.

Lemma 8. Under the notation above, the following hold:

i) Let $x$ be an element of $M^{\sharp} / M$. If $h(x, y)$ is trivial for all $y \in M^{\sharp} / M$, then $x=0$.

ii) Assume $\mathrm{M}$ is an h-module over $\mathrm{C}$. Let $\mathrm{V}_{1} \perp \mathrm{V}_{2}$ be a decomposition of $\mathrm{M}_{K}$ and let $p_{i}$ be the projection of $\mathrm{M}_{K}$ on $\mathrm{V}_{i}$. Let $M_{i}$ be the C-ñ-modules $\mathrm{M} \cap \mathrm{V}_{i}$. Then

$\circ M_{i}^{\sharp}=p_{i}(M)$,

$\circ \operatorname{Res}\left(\mathrm{M}_{1} \perp \mathrm{M}_{2}\right)$ contains $I=M /\left(M_{1} \perp M_{2}\right)$, and $I$ is self orthogonal,

$\circ$ the projections $M_{1}^{\sharp} / M_{1} \stackrel{p_{1}}{\longleftarrow} I \stackrel{p_{2}}{\longrightarrow} M_{2}^{\sharp} / M_{2}$ are isomorphisms (of $C$-modules) and induce an isomorphism $\operatorname{Res}\left(\mathrm{M}_{1}\right) \simeq\langle-1\rangle \otimes \operatorname{Res}\left(\mathrm{M}_{2}\right)$.

Recall that property $\mathcal{A}(\mathrm{M}, \mathrm{N}, \alpha, \beta)$ over $\mathrm{C}$ (in the presence of property $\mathcal{C}(\mathrm{M}, \mathrm{N})$ ) says that for $n \geq \alpha k+\beta$, the orthogonal complement in $\mathrm{M} \perp \mathrm{N}^{\perp n}$ of any $\tilde{\mathrm{h}}$-submodule $\mathrm{Q}$ of rank no larger than $(3 k+2) \nu$ contains a copy of $\mathrm{N}$. The lemma above shows that the following property implies $\mathcal{A}(\mathrm{M}, \mathrm{N}, \alpha, \beta)$ :

$\mathcal{A}^{\prime}(\mathrm{M}, \mathrm{N}, \alpha, \beta)$ : For all $n \geq \alpha k+\beta$, any $\tilde{\mathrm{h}}$-submodule $\mathrm{P}$ of $\mathrm{M} \perp \mathrm{N}^{\perp n}$ of rank at least $\mu+(n-3 k-2) \nu$, whose residue $\operatorname{Res}(\mathrm{P})$ admits a system of $l$ generators (over $\mathrm{C}$ ) with $l \leq(2 k+2) \nu$, represents $\mathrm{N}$.

\subsection{Properties $\mathcal{C}$ and $\mathcal{A}$ when $R$ is a local ring.}


2.4.1. $R$ a local ring and $C$ of type D2S, D2NSI, D4, or $R$ a non-dyadic local ring.

Let $R$ be a local ring with maximal ideal $\mathfrak{p}$ and uniformiser $\pi_{R}$. Let $\mathrm{C}=(C, R)$ be a composition algebra over $R$. Let $\mathrm{M}$ be an $\tilde{\mathrm{h}}$-module. In the situations studied in this paragraph, $\mathrm{M}$ admits a canonical form. More precisely

i) If $R$ is non-dyadic and $\mathrm{C}$ is of type $\mathrm{D} 1$, there exists a unique sequence of integers $n_{i}$ and a unique sequence of elements $\varepsilon_{i} \in R^{\times} / N\left(C^{\times}\right)$for which one has a decomposition

$$
\mathrm{M} \simeq \perp_{i}\left\langle\pi_{R}^{i}\right\rangle \otimes \mathrm{I}_{n_{i}}^{\varepsilon_{i}}(\mathrm{C}) .
$$

ii) If $\mathrm{C}$ is of type D2S, D4S, D2NSI, there exists a unique sequence of integers $n_{i}$ for which one has a decomposition

$$
\mathrm{M} \simeq \perp_{i}\left\langle\pi_{R}^{i}\right\rangle \otimes \mathrm{I}_{n_{i}}(\mathrm{C})
$$

iii) If $\mathrm{C}$ is of type D4NS, or if $R$ is non-dyadic and $\mathrm{C}$ is of type D2NSR, with uniformiser $\pi_{C}$, then there exists a unique sequence of integers $n_{i}$, and a unique sequence of elements $\varepsilon_{i} \in R^{\times} / N\left(C^{\times}\right)$for which one has a decomposition

$$
\mathrm{M} \simeq \sum_{i} \mathrm{M}_{i} \quad \text { with } \begin{cases}\mathrm{M}_{i}=\left\langle\pi_{R}^{i / 2}\right\rangle \otimes \mathrm{I}_{n_{i}}^{\varepsilon_{i}}(\mathrm{C}) & \text { if } \quad i \text { is even } \\ \mathrm{M}_{i}=\left(\mathrm{H}^{\left(\pi_{C}^{i}\right)}(\mathrm{C})\right)^{\perp n_{i}} & \text { if } \quad i \text { is odd }\end{cases}
$$

Proofs of these results may be found in [O'Meara 92:11 \& 92:2] for i), in [S1964 3.1] for ii)D2S, in [S1964 6.11] for ii)D4S, in [J1962 7] for ii) D2NSI, in [J1962 6.1] for iii)D4NS and in [J1962 8.1] for iii)D2NSR. We immediately deduce from these facts and from lemma 8 :

Proposition 9. Properties $\mathcal{C}$ and $\mathcal{A}^{\prime}(5,6)$ hold over all composition algebras $\mathrm{C}$ considered above.

\subsection{2. $R$ a dyadic local ring and $\mathrm{C}$ is of type D1 or D2NSR.}

In this situation, there are no canonical forms, at least not as simple as above. Moreover, property $\mathcal{C}$ does not hold in general. Nevertheless, the following hold:

i) Assume $\mathrm{N}$ is hyperbolic. Then for all h-modules $\mathrm{M}$, property $\mathcal{C}(\mathrm{M}, \mathrm{N})$ holds.

ii) Let $\mathrm{M}$ be an h-module. If $\mathrm{C}$ is of type $\mathrm{D} 1$ and if $\operatorname{rk}(\mathrm{M}) \geq 5$, or if $\mathrm{C}$ is of type D2NSR and if $\operatorname{rk}(\mathrm{M}) \geq 3$, then $\mathrm{M}$ represents a hyperbolic plane.

Proofs may be found in [O'Meara 93:14 \& 93:18] for the D1 case and in [J1962 9.3 \& 10.3] for the D2NSR case.

Proposition 10. Let $R$ be a dyadic local ring, $\mathrm{C}$ a composition algebra over $R$ and $\mathrm{M}$ and $\mathrm{N}$ two h-modules over $\mathrm{C}$. If $\mathrm{N}$ is hyperbolic, then

○ property $\mathcal{C}(\mathrm{M}, \mathrm{N})$ holds,

○ if $\mathrm{C}$ is of type D1, then property $\mathcal{A}^{\prime}(\mathrm{M}, \mathrm{N}, 5,7)$ holds,

○ if $\mathrm{C}$ is of type D2NSR, then property $\mathcal{A}^{\prime}(\mathrm{M}, \mathrm{N}, 5,6)$ holds.

We will have to use the following consequence of proposition 10.

Proposition 11. Let $R$ be a dyadic local ring, let $\mathrm{C}$ be a composition algebra of type D1 (resp. D2NS) over $R$ and let $\mathrm{M}$ and $\mathrm{N}$ be two h-modules over $\mathrm{C}$. If $\mathrm{N}$ is hyperbolic, and $n \geq 8$ (resp. $n \geq 7$ ), then the group $\mathrm{U}\left(\mathrm{M} \perp \mathrm{N}^{\perp n}\right)$ is generated by conjugates of the subgroup $\mathrm{U}\left(\mathrm{M} \perp \mathrm{N}^{\perp 7}\right)$ (resp. $\left.\mathrm{U}\left(\mathrm{M} \perp \mathrm{N}^{\perp 6}\right)\right)$.

\subsection{The case of a ring of $(S)$-integers.}




\subsubsection{The rings to be considered.}

- $F$ is a number field with ring of integers $\mathcal{O}$, and $\mathcal{V}^{f}$ (resp. $\mathcal{V}^{\infty}$ ) is its set of non-archimedean (resp. archimedean) places and $\mathcal{V}$ is the union $\mathcal{V}=\mathcal{V}^{\infty} \cup \mathcal{V}^{f}$. For $\mathfrak{p}$ in $\mathcal{V}$, the field $F_{\mathfrak{p}}$ is the completion of $F$ with respect to $\mathfrak{p}$, and for $\mathfrak{p} \in \mathcal{V}^{f}$ (resp. $\left.\mathfrak{p} \in \mathcal{V}^{\infty}\right)$, the ring $\mathcal{O}_{\mathfrak{p}}$ is the ring of integers in $F_{\mathfrak{p}}$ (resp. we put $\mathcal{O}_{\mathfrak{p}}=F_{\mathfrak{p}}$ ).

- $S$ is a subset of $\mathcal{V}$ with finite complement $\Omega$ containing $\mathcal{V}^{\infty}$ and at least one non-archimedean place $\mathfrak{p}_{0}$, and $S_{2}$ is the subset of dyadic elements of $S$.

- $A$ is the ring of $S$-integers in $F: A=F \cap \bigcap_{\mathfrak{p} \in S} \mathcal{O}_{\mathfrak{p}}$, and, for any $\mathfrak{p} \in \mathcal{V}$, we put $A_{\mathfrak{p}}=A \otimes_{\mathcal{O}} \mathcal{O}_{\mathfrak{p}}$.

- $\mathbb{A}$ is the product $\prod_{\mathfrak{p} \in \mathcal{V}} A_{\mathfrak{p}}$ and $\mathbb{F}$ is the tensor product $F \otimes_{A} \mathbb{A}$.

- $\mathrm{C}=(C, N)$ is a composition algebra over $A$ of dimension $d$.

\subsubsection{The genus and the $\Omega$-genus of an $\tilde{\mathrm{h}}$-module.}

- Let $\mathrm{V}=(V, h)$ be a hermitian module over a composition algebra $\mathrm{D}=(D, n)$ over a field $R$ where 2 is invertible. We define a subgroup $\mathrm{U}^{\prime}(\mathrm{V})$ of $\mathrm{U}(\mathrm{V})$ as follows.

$\circ$ if $\mathrm{D}$ is 4-dimensional, then we put $\mathrm{U}^{\prime}(\mathrm{V})=\mathrm{U}(\mathrm{V})$,

$\circ$ if $\mathrm{D}$ is 2-dimensional, then the determinant $\delta$ is defined on $\mathrm{U}(\mathrm{V})$, and we put $\mathrm{U}^{\prime}(\mathrm{V})=\mathrm{SU}(\mathrm{V})=\operatorname{ker}(\delta)$; the quotient $\mathrm{U}(\mathrm{V}) / \mathrm{U}^{\prime}(\mathrm{V})$ is identified via $\delta$ with the abelian group

$$
\mathrm{S}^{1}\left(\mathrm{C}_{F}\right):=\{x \in F, \quad x \bar{x}=1\}
$$

$\circ$ if $\mathrm{D}$ is 1-dimensional, then $\mathrm{U}(\mathrm{V})$ is generated by reflections $\tau_{x}: y \mapsto y-2 \frac{h(x, y)}{h(x, x)} x$ (with $x$ non-isotropic). On $\mathrm{U}(\mathrm{V})$ is defined the spinor norm $\Theta: \mathrm{U}(\mathrm{V}) \rightarrow R^{\times} / R^{\times 2}$, characterized by $\Theta\left(\tau_{x}\right)=h(x, x)$. The subgroup $\mathrm{U}^{\prime}(\mathrm{V})$ is then the kernel of $\Theta$ restricted to $\mathrm{SU}(\mathrm{V})$. It is also the image of the natural morphism $\mathrm{Spin}(\mathrm{V}) \rightarrow$ $\mathrm{U}(\mathrm{V})$.

For a subset $E$ of $\mathrm{V}$, and $G=\mathrm{U}(\mathrm{V})$ (resp. $G=\mathrm{U}^{\prime}(\mathrm{V})$ ), we denote by $\mathrm{U}(E)$ (resp. $\mathrm{U}^{\prime}(E)$ ) the set of elements $\gamma \in G$ such that $E \cdot \gamma=E$. For a group morphism $\varphi: \mathrm{U}(\mathrm{V}) \rightarrow H$ and a subset $E$ of $\mathrm{V}$, we put $\varphi(E)=\varphi(\mathrm{U}(E))$.

- Let $\mathrm{V}=(V, h)$ be an h-module over $\mathrm{C}_{F}$ of rank $n$. Let $M$ be a $C$-lattice on $\mathrm{V}$. The genus gen $(M)$ of $M$ is the set of all $C$-lattices $M^{\prime}$ on $\mathrm{V}$ such that $M$ and $M^{\prime}$ are isometric at each place $\mathfrak{p}$ (i.e. there exists an element $\gamma_{\mathfrak{p}}$ of $\mathrm{U}\left(\mathrm{V}_{\mathfrak{p}}\right)$ such that $M_{\mathfrak{p}}^{\prime}=M_{\mathfrak{p}} \cdot \gamma_{\mathfrak{p}}$ for each $\mathfrak{p}$; note that for almost all $\mathfrak{p}$, one has an equality $M_{\mathfrak{p}}=M_{\mathfrak{p}}^{\prime}$ so that $\gamma_{\mathfrak{p}}$ lies in $\left.\mathrm{U}\left(M_{\mathfrak{p}}\right)\right)$.

On gen $(M)$, we define the following equivalence relation: $M^{\prime} \sim M^{\prime \prime}$ if $M_{\mathfrak{p}}^{\prime}$ and $M_{\mathfrak{p}}^{\prime \prime}$ are isometric via an element of $\mathrm{U}^{\prime}\left(\mathrm{V}_{\mathfrak{p}}\right)$ at each place $\mathfrak{p}$. The set of equivalence classes for $\sim$ is written $\Omega$ gen $(M)$. (A justification for this notation is that the inclusion of the commutator subgroup $\Omega U(\mathrm{~V})$ in $\mathrm{U}^{\prime}(\mathrm{V})$ is almost always an equality.) Note that the group $\mathrm{U}(\mathrm{V})$ still acts on $\Omega \operatorname{gen}(M)$. Let $\overline{\operatorname{\Omega gen}}(M)$ be the quotient of $\Omega$ gen $(M)$ by this action. We denote by $\overline{M^{\prime}}$ the class of an element $M^{\prime}$ in this quotient. Then

$\circ$ The set $\operatorname{gen}(M)$ is parametrized by the quotient $\mathrm{U}\left(M_{\mathbb{A}}\right) \backslash \mathrm{U}\left(\mathrm{V}_{\mathbb{F}}\right)$.

$\circ$ The set $\Omega \operatorname{gen}(M)$ is parametrized by the quotient $\mathrm{U}\left(M_{\mathbb{A}}\right) \backslash \mathrm{U}\left(\mathrm{V}_{\mathbb{F}}\right) / \mathrm{U}^{\prime}\left(\mathrm{V}_{\mathbb{F}}\right)$.

$\circ$ The set $\overline{\operatorname{\Omega gen}}(M)$ is parametrized by the quotient

$$
Q(M):=\mathrm{U}\left(M_{\mathbb{A}}\right) \backslash \mathrm{U}\left(\mathrm{V}_{\mathbb{F}}\right) /\left[\mathrm{U}^{\prime}\left(\mathrm{V}_{\mathbb{F}}\right) \cdot \mathrm{U}(\mathrm{V})\right]
$$

This is an abelian group, that acts simply transitively on $\overline{\Omega g e n}(M)$. This action is natural in the following sense: if $M(\operatorname{resp} . N)$ is an $A$-lattice in an h-module $\mathrm{V}$ (resp. W) over $\mathrm{C}_{F}$, then the map

$$
\begin{aligned}
& a(M, N): \overline{\operatorname{\Omega gen}}(M) \rightarrow \overline{\operatorname{\Omega gen}}(M \perp N) \\
& M^{\prime} \quad \mapsto \quad M^{\prime} \perp N
\end{aligned}
$$

is equivariant with respect to the morphism $Q(M) \rightarrow Q(M \perp N)$. 
Proposition 12. Let $M$ (resp. $N$ ) be a C-lattice on an $\mathrm{h}$-module $\mathrm{V}=(\mathrm{V}, h)$ (resp. $\mathrm{W}=\left(W, h^{\prime}\right)$ ) over $\mathrm{C}_{F}$. If $\mathrm{C}$ is of type D1 (resp. D2 or D4), assume $\mathrm{V}$ has dimension greater than 3 (resp. 1). Then the map $a(M, N)$ is onto.

Proof: First, note that if $\mathrm{C}_{F}$ is of type $\mathrm{D} 4$, then there is nothing to prove. When $\mathrm{C}_{F}$ is of type $\mathrm{D} 2$, the isomorphism

$$
Q(M) \simeq \delta\left(M_{\mathbb{A}}\right) \backslash \mathrm{S}^{1}\left(\mathrm{C}_{\mathbb{F}}\right) / \mathrm{S}^{1}\left(\mathrm{C}_{F}\right)
$$

immediately implies the result. When $\mathrm{C}_{F}$ is of type $\mathrm{D} 1$, the natural map $\mathrm{SU}\left(M_{A}\right) \backslash \mathrm{SU}\left(\mathrm{V}_{\mathbb{F}}\right) \rightarrow \mathrm{U}\left(M_{A}\right) \backslash \mathrm{U}\left(\mathrm{V}_{\mathbb{F}}\right)$ is onto. This furnishes an epimorphism $\Theta\left(M_{\mathbb{A}}\right) \backslash \Theta\left(\mathrm{V}_{\mathbb{F}}\right) / \Theta(\mathrm{V}) \rightarrow Q(M)$. The result is then implied by the fact that $\Theta\left(V_{\mathbb{F}}\right) \rightarrow \Theta\left(V_{\mathbb{F}} \perp W_{\mathbb{F}}\right)$ is onto, which follows from the equality $\Theta\left(V_{\mathfrak{p}}\right)=F_{\mathfrak{p}}^{\times}$, that is valid at any non-archimedean place $\mathfrak{p}$ (under the assumption $\operatorname{dim}(V) \geq 3$, see [O'Meara 91.6]).

The following result follows from Kneser's strong approximation theorem [K 1966] (see [O'Meara 104:4] for the D1 case).

Theorem 13. Let $M$ be a $\mathrm{C}$-lattice in an $\mathrm{h}$-module $\mathrm{V}$ over $C_{F}$ of rank $n>1$. If $\mathrm{C}$ is of type D1, assume $n \geq 5$. If $\mathrm{V}_{\mathfrak{p}}$ is isotropic for some $\mathfrak{p} \in \Omega$, then two lattices $M^{\prime}$ and $M^{\prime \prime}$ in $\operatorname{gen}(M)$ have the same image in $\overline{\Omega \operatorname{gen}}(M)$ if and only if they are isometric via an element of $\mathrm{U}(\mathrm{V})$.

\subsubsection{Properties $\mathcal{C}$ and $\mathcal{A}$.}

An h-module $\mathrm{M}$ is said to be locally cancellable if it is hyperbolic at each place $\mathfrak{p} \in S_{2}$ (so that $\mathcal{C}\left(\mathrm{L}_{\mathfrak{p}}, \mathrm{M}_{\mathfrak{p}}\right.$ ) holds for any $\tilde{h}$-module $L$ over $\mathrm{C}$, at each place $\mathfrak{p}$ ).

An h-module $\mathrm{M}$ is said to be initial if it is at least of rank $4-d$ over $\mathrm{C}$, if there exists a place $\mathfrak{p} \in \Omega$ such that $\mathrm{M}_{\mathfrak{p}}$ be isotropic, and if $a(\mathrm{M}, \mathrm{N})$ is bijective for each locally cancellable h-module $\mathrm{N}$.

Proposition 14. Let $\mathrm{M}$ be an initial $\mathrm{h}$-module over $\mathrm{C}$, let $\mathrm{N}$ be a locally cancellable $\mathrm{h}$-module over $\mathrm{C}$, and let $\mathrm{P}$ be any sub-h-module of $\mathrm{M} \perp \mathrm{N}$ such that there exists an isomorphism $\mathrm{P} \simeq \mathrm{N}$. Then the modules $\mathrm{P}^{\perp}$ and $\mathrm{M}$ are isomorphic.

Proof: Let $\mathrm{R}$ be the orthogonal complement of $\mathrm{P}$ in $\mathrm{M} \perp \mathrm{N}$, and put $\mathrm{V}=\mathrm{R}_{F}\left(\operatorname{resp} \mathrm{W}=(\mathrm{M})_{F}\right)$. Since $\mathrm{N}$ is locally cancellable, there exists an isomorphism $R_{\mathfrak{p}} \simeq(\mathrm{M})_{\mathfrak{p}}$ at each place $\mathfrak{p}$. In particular, the Hasse principle shows that $\mathrm{V}$ and $\mathrm{W}$ are isomorphic. So we can consider $R$ and $M$ as $C$-lattices in $\mathrm{V}$, that are in the same genus. Now since the map $a(M, N)$ is bijective, $R$ and $M$ are in the same class in $\overline{\Omega g e n}(M)$. It follows from theorem 13 that $R$ and $M \perp N$ are isometric.

Proposition 15. Let $\mathrm{M}$ be an $\tilde{\mathrm{h}}$-module over $\mathrm{C}$ that is isotropic at some place $\mathfrak{p} \in \Omega$ and let $\mathrm{N}$ be a locally cancellable h-module over $\mathrm{C}$ such that $\mathrm{M}_{\mathfrak{p}}$ represent $\mathrm{N}_{\mathfrak{p}}$ at each place $\mathfrak{p}$. Assume the inequality $\operatorname{rk}(M) \geq$ $\operatorname{rk}(N)+4-d$ holds. If $\mathrm{C}$ is of type D1, assume moreover that $M$ has rank at least 5 . Then the module $\mathrm{M}$ represents $\mathrm{N}$.

Proof: Let us write $\mathrm{V}=\mathrm{M}_{F}$ and $\mathrm{W}=\mathrm{N}_{F}$. By the Hasse principle, we can think of $\mathrm{W}$ as a sub-h-module of $\mathrm{V}$. Let $\mathrm{X}$ be the orthogonal complement of $\mathrm{W}$ in $\mathrm{V}$. For each $\mathfrak{p} \in \mathcal{V}$, let $\mathrm{N}(\mathfrak{p})$ be any representation of $\mathrm{N}_{\mathfrak{p}}$ in $\mathrm{M}_{\mathfrak{p}}$, and let $\mathrm{L}(\mathfrak{p})$ be the orthogonal complement of $\mathrm{N}(\mathfrak{p})$ in $\mathrm{M}_{\mathfrak{p}}$. Let $L^{\prime}$ be any $A$-lattice in $\mathrm{X}$. Then at all places, there is an equality $M_{\mathfrak{p}}=L(\mathfrak{p}) \perp N(\mathfrak{p})$, and at almost all places, there is an equality $M_{\mathfrak{p}}=\left(L^{\prime} \perp N\right)_{\mathfrak{p}}$. In particular, since $\mathrm{N}$ is locally cancellable, at almost all places, there is an isometry $\mathrm{L}_{\mathfrak{p}}^{\prime} \simeq \mathrm{L}(\mathfrak{p})$. From [O'Meara 81:14] it follows that there exists a lattice $L$ on X such that $L_{\mathfrak{p}}$ and $L(\mathfrak{p})$ are isometric at each place $\mathfrak{p}$. Finally, the map $a(L, N)$ is onto by proposition 12, and this together with theorem 13 proves the proposition.

Corollary 16. Let $\mathrm{M}$ be an initial $\mathrm{h}$-module over $A$ and let $\mathrm{N}$ be a locally cancellable $\mathrm{h}$-module over $A$ of rank $\nu$. Then properties $\mathcal{C}(\mathrm{M}, \mathrm{N})$ and $\mathcal{A}(\mathrm{M}, \mathrm{N}, \alpha, \beta)$ hold with $\alpha=\max (5,2+\nu)$ and $\beta=\max (10,5+\nu)$.

Proof: From 2.4 it follows that if $n \geq 5 k+10$ (resp. $n \geq(\nu+2) k+(\nu+5)$ ), then $\mathrm{Q}_{\mathfrak{p}}^{\perp}$ represents $\mathrm{N}_{\mathfrak{p}} \perp \mathrm{U}(\mathfrak{p})$ at each non-archimedean place $\mathfrak{p}$ (resp. at each archimedean place $\mathfrak{p}$ ) with $\mathrm{U}(\mathfrak{p})$ of rank at least 4. It follows that $Q^{\perp}$ and $N$ satisfy the hypotheses of proposition 15 since $Q_{\mathfrak{p}_{0}}$ is at least 5-dimensional and hence isotropic. 


\subsection{The proof of the theorem in the general case.}

Theorem 17. Let $R$ be a ring, $\mathrm{C}$ a composition algebra over $R, \mathrm{M}$ and $\mathrm{N}$ two h-modules over $\mathrm{C}$. Then in the following cases, property $\mathrm{P}(\mathrm{M}, \mathrm{N}, f)$ holds with $f_{k}=\alpha k+\beta$ :

\begin{tabular}{|c|c|c|}
\hline$R$ & $\alpha$ & $\beta$ \\
\hline $\mathbb{C}$ & 3 & 1 \\
\hline $\mathbb{R}$ & $\nu+2$ & $\nu+2$ \\
\hline finite field of odd char. & 3 & 2 \\
\hline finite field of char. 2 & 6 & 10 \\
\hline local field & 3 & 4 \\
\hline global number field & $\nu+2$ & $\max (\nu+2,6)$ \\
\hline non-dyadic local ring & 5 & 6 \\
\hline dyadic local ring & 40 & 64 \\
\hline ring of S-integers & $16+64 \nu$ & $69+64 \nu$ \\
\hline
\end{tabular}

We will use the following lemmas.

Lemma 18. Let $R$ be a local ring, $\mathrm{C}=(C, N)$ a composition algebra over $R$ and $\mathrm{M}$ an $\mathrm{h}$-module over $\mathrm{C}$.

a) If $R$ is a local field, or a non-dyadic local ring, and if $\mathrm{C}$ is 1-dimensional, then $\mathrm{M}^{\perp 4}$ is hyperbolic.

b) If $R$ is a local field, or a non-dyadic local ring, and if $\mathrm{C}$ is 2-dimensional or 4-dimensional, then $\mathrm{M}^{\perp 2}$ is hyperbolic.

c) If $R$ is a dyadic local ring and if $\mathrm{C}$ is of type D2S, D2NSI, or D4, then $M^{\perp 2}$ is hyperbolic.

d) If $R$ is a dyadic local ring and $C$ is of any type, there exists an isomorphism $\mathrm{M}^{\perp 9} \simeq \mathrm{E} \perp \mathrm{M}$ with $E$ hyperbolic.

Proof: Of course, if $\mathrm{M}$ is the trivial h-module, everything is true. Assume M is non-trivial.

a) In the case of local fields, it suffices to prove the result for a 1-dimensional $\mathrm{M}=<\alpha>$. Then $\mathrm{M}^{\perp 8}$ is 8-dimensional, has determinant $\alpha^{8}=1 \in R^{\times} / R^{\times 2}$ and Hasse invariant $(\alpha, \alpha)^{28}=1 \in\{ \pm 1\}$. So $\mathrm{M}^{\perp 8}$ is isomorphic to $\mathrm{H}(R)^{4}$ since these modules have the same invariants. In the case of a non-dyadic local ring, we know from 2.4.1 i) that all unimodular lattices on $\mathrm{M}_{K}$ are isomorphic ; since $\mathrm{M}_{K}^{\perp 8}$ is hyperbolic, it supports a hyperbolic unimodular lattice. The proofs of b) and c) are the same, mutatis mutandis.

d) The existence of $\sqrt{-7}$ in $\mathbb{Z}_{2}$ furnishes an isomorphism $\mathrm{I}_{4}\left(\mathbb{Z}_{2}\right) \simeq\langle-1\rangle \otimes \mathrm{I}_{4}\left(\mathbb{Z}_{2}\right)$. Moreover, there exists an isomorphism $\mathrm{I}_{3}^{(-1)}\left(\mathbb{Z}_{2}\right) \simeq \mathrm{H}\left(\mathbb{Z}_{2}\right) \perp \mathrm{I}_{1}\left(\mathbb{Z}_{2}\right)$. We deduce an isomorphism $\mathrm{I}_{9}\left(\mathbb{Z}_{2}\right) \simeq \mathrm{H}\left(\mathbb{Z}_{2}\right)^{\perp 4} \perp \mathrm{I}_{1}\left(\mathbb{Z}_{2}\right)$ which implies, once tensored with $\mathrm{M}$, an isomorphism $\mathrm{M}^{\perp 9} \simeq \mathrm{E} \perp \mathrm{M}$ with $\mathrm{E}=\mathrm{H}^{\perp 4}\left(\mathbb{Z}_{2}\right) \otimes_{\mathbb{Z}_{2}} \mathrm{M}$ hyperbolic.

Lemma 19. Let $\mathrm{M}$ be an $\mathrm{h}$-module over $\mathrm{C}$ that is isotropic at some place $\mathfrak{p} \in \Omega$ and let $\mathrm{N}$ be any hmodule over $\mathrm{C}$. There exists an isomorphism $\mathrm{M} \perp \mathrm{N}^{\perp 9} \simeq \mathrm{M} \perp \mathrm{N} \perp \mathrm{E}$ with $\mathrm{E}$ locally cancellable, and then $\mathrm{M} \perp \mathrm{N}^{u} \perp \mathrm{E} \perp \mathrm{E} \simeq \mathrm{M} \perp \mathrm{N}^{\perp 16+u}$ is initial for $u \geq 1$.

Proof: We have seen that there exists, at any dyadic place $\mathfrak{p}$ a hyperbolic lattice $H(\mathfrak{p})$ on $\mathrm{W}_{\mathfrak{p}}^{\perp 8}$ such that $H(\mathfrak{p}) \perp N_{\mathfrak{p}}$ and $N_{\mathfrak{p}}^{\perp 9}$ are isometric. Let $E$ be a lattice on $\mathrm{W}^{\perp 8}$ such that $E_{\mathfrak{p}}$ is isometric to $H(\mathfrak{p})$ if $\mathfrak{p}$ is an element of $\mathcal{S}_{2}$, and such that $E_{\mathfrak{p}}$ is isometric to $N_{\mathfrak{p}}^{\perp 8}$ if $\mathfrak{p}$ is an element of $\mathcal{V}-\mathcal{S}_{2}$ (note that $N_{\mathfrak{p}}^{\perp 8}$ is hyperbolic if $\mathfrak{p}$ is in $\mathcal{V}^{f}-\mathcal{S}_{2}$ ). Such a lattice exists, see [O'Meara 81:14], and is locally cancellable. Since $E \perp N$ and $N^{\perp 9}$ are in the same genus and the natural map $Q(E) \rightarrow Q(M \perp N \perp E)$ is onto, it follows from theorem 13 that $E$ can be chosen so that $M \perp E \perp N$ and $M \perp N^{\perp 9}$ are isometric. That $M \perp N \perp E \perp E$ is initial follows directly from 11 .

Proof of the theorem: recall from the introduction that in order that $\mathrm{P}\left(\mathrm{M}^{\prime}, \mathrm{N}^{\prime}, \alpha, \beta\right)$ holds, it suffices that $\mathcal{C}\left(\mathrm{M}^{\prime}, \mathrm{N}^{\prime}\right)$ and $\mathcal{A}\left(\mathrm{M}^{\prime}, \mathrm{N}^{\prime}\right)$ hold. Now assume $\mathrm{P}\left(\mathrm{M}^{\prime}, \mathrm{N}^{\prime}, \alpha, \beta\right)$ holds with $\mathrm{M}^{\prime} \simeq \mathrm{M} \perp \mathrm{N}^{s}$ and $\mathrm{M}^{\prime} \perp\left(\mathrm{N}^{\prime}\right)^{\perp n} \simeq \mathrm{M} \perp$ $\mathrm{N}^{\perp s+n t}$ for a given $t$ and all $s \geq s_{0}$. Then, since the morphism $i\left(\mathrm{M}^{\prime}, \mathrm{N}^{\prime}, n\right)$ factorizes through $\mathrm{U}\left(\mathrm{M}^{\prime} \perp \mathrm{N} \perp \mathrm{N}^{\prime}\right)$, one easily checks that $\mathrm{P}\left(\mathrm{M}, \mathrm{N}, \alpha t, s_{0}+(\beta+1) t-1\right)$ holds. In the case of an $(S)$-arithmetic ring, for example if 
(M, N) is indefinite, then $\mathrm{M} \perp \mathrm{N}^{\perp 5}$ is isotropic at some place $\mathfrak{p}$, and it follows from lemma 19 that $\mathrm{M}^{\prime}=\mathrm{M} \perp$ $\mathrm{N}^{s}$ is initial for $s \geq 22$, and that there exists a locally cancellable $\mathrm{N}^{\prime}$ such that $\mathrm{M}^{\prime} \perp\left(\mathrm{N}^{\prime}\right)^{\perp n} \simeq \mathrm{M} \perp \mathrm{N}^{s+8 n}$. Let $\nu$ be the rank of $\mathrm{N}$. Corollary 16 implies that properties $\mathcal{C}\left(\mathrm{M}^{\prime}, \mathrm{N}^{\prime}\right)$ and $\mathcal{A}\left(\mathrm{M}^{\prime}, \mathrm{N}^{\prime}, 2+8 \nu, 5+8 \nu\right)$ hold. It follows that $\mathrm{P}(\mathrm{M}, \mathrm{N}, \alpha, \beta)$ holds with $\alpha=16+64 \nu$ and $\beta=69+64 \nu$.

\section{The CASE OF $\mathrm{O}_{n}\left(\mathbb{Z}\left[\frac{1}{2}\right]\right)$}

We shall now concentrate on the case $R=\mathbb{Z}\left[\frac{1}{2}\right]$, and consider the algebraic group $\mathrm{O}_{n}$ defined over $\mathbb{Z}\left[\frac{1}{2}\right]$ by $\mathrm{O}_{n}(k)=\mathrm{U}\left(\mathrm{I}_{n}(k)\right)$.

\subsection{The stability theorem in the special case.}

Proposition 20. Let $\mathrm{M}$ and $\mathrm{N}$ be $\tilde{\mathrm{h}}$-modules over $R$. Assume that at each place $\mathfrak{p}, \mathrm{M}_{\mathfrak{p}}$ represents an $\mathrm{h}$ module of rank 2 , and that $M$ is at least of rank 5. Then there exists an isomorphism $\mathrm{M} \simeq \mathrm{N}$ iff $\mathrm{M}_{\mathfrak{p}}$ and $\mathrm{N}_{\mathfrak{p}}$ are isomorphic at each place $\mathfrak{p}($ of $\mathbb{Q})$.

Proof: We may consider $M$ and $N$ as lattices in the same genus in $\mathrm{V}:=\mathrm{M}_{\mathbb{Q}}$. From the hypothesis on $\mathrm{M}$ and the fact that $\mathbb{Z}\left[\frac{1}{2}\right]$ is a principal ideal ring it follows that the group $Q(M)$ is trivial. Theorem 13 then implies the proposition.

Corollary 21. Let $\mathrm{M}$ be an n-dimensional positive definite $\mathrm{h}$-module over $R$. Then there exists an isomorphism $\mathrm{M} \simeq \mathrm{I}_{n}^{(d)}$ with $d \in\{1,2\}$. In particular, property $\mathcal{C}\left(\mathrm{M}, \mathrm{I}_{1}\right)$ holds for all positive definite $\mathrm{h}$-modules $M$ over $R$.

Proof: this follows from the proposition when $n \geq 5$. For $n \leq 4$, it is already true that a positive definite $\tilde{h}$-module of determinant $d \in\{1,2\}$ is isomorphic to $\mathrm{I}_{n}^{(d)}(\mathbb{Z})$ (see [K1957]).

Proposition 22. Let $\mathrm{M}$ be an $\tilde{\mathrm{h}}-$ module of rank at least 5 over $R$ and let d be either 1 or 2 ; if $\mathrm{M}_{\mathfrak{p}}$ represents $\mathrm{I}_{1}^{(d)}$ at each place $\mathfrak{p}$, then $\mathrm{M}$ represents $\mathrm{I}_{1}^{(d)}$. In particular, property $\mathcal{A}\left(0, \mathrm{I}_{1}, \max (3 k+4,2 k+6)\right)$ holds.

Corollary 23. Property $\mathrm{P}\left(0, \mathrm{I}_{1}, \max (3 k+4,2 k+6)\right)$ holds.

\subsection{On the structure of the groups $\mathrm{O}_{n}\left(\mathbb{Z}\left[\frac{1}{2}\right]\right)$.}

3.2.1. $\mathrm{O}_{n}\left(\mathbb{Z}\left[\frac{1}{2}\right]\right)$ is generated by reflections $(n \geq 1)$.

Let us denote by $\mathrm{I}_{n}(\mathbb{Z}) \subset \mathrm{I}_{n}\left(\mathbb{Z}\left[\frac{1}{2}\right]\right)$ the $\mathbb{Z}$-lattice whose vectors have integral coefficients in the canonical basis. We put

$$
\mathrm{D}_{4} \perp \mathrm{I}_{n-4}(\mathbb{Z}):=\left\{x \in \mathrm{I}_{n}(\mathbb{Z}), x_{1}+\cdots+x_{4} \equiv 0 \bmod 2\right\} .
$$

Note that there are inclusions $c_{n}: \mathrm{O}_{n}(\mathbb{Z}) \subset \mathrm{O}_{n}\left(\mathbb{Z}\left[\frac{1}{2}\right]\right)$, and $d_{n}: \operatorname{Aut}\left(\mathrm{D}_{4} \perp \mathrm{I}_{n-4}(\mathbb{Z})\right)=\operatorname{Aut}\left(\mathrm{D}_{4}\right) \times \mathrm{O}_{n-4}(\mathbb{Z}) \subset$ $\mathrm{O}_{n}\left(\mathbb{Z}\left[\frac{1}{2}\right]\right)$.

$\circ n \leq 3$ : The inclusion $c_{n}$ is an equality. This follows immediately from the remark that the set of vectors of length 1 in $\mathrm{I}_{n}(\mathbb{Z})$ and the set of vectors of length 1 in $\mathrm{I}_{n}\left(\mathbb{Z}\left[\frac{1}{2}\right]\right)$ coincide for these values of $n$.

$\circ n=4$ : The inclusion $d_{n}$ is an equality. This follows from the well known fact that $\mathrm{D}_{4}^{\sharp}$ is the set of integral vectors in $\mathrm{I}_{4}\left(\mathbb{Z}\left[\frac{1}{2}\right]\right)$.

$\circ n \geq 5$ : Let $Y_{n}$ be the geometric realization of the poset of $\mathbb{Z}$-lattices on $\mathrm{I}_{n}\left(\mathbb{Z}\left[\frac{1}{2}\right]\right)$ which are either isometric to $\mathrm{I}_{n}(\mathbb{Z})$ or to $\mathrm{D}_{4} \perp \mathrm{I}_{n-4}(\mathbb{Z})$ (ordered by inclusion). This is a is a graph acted upon by $\mathrm{O}_{n}\left(\mathbb{Z}\left[\frac{1}{2}\right]\right.$ ) with fundamental domain

$$
\mathrm{D}_{4} \perp \mathrm{I}_{n-4}(\mathbb{Z}) \subset \mathrm{I}_{n}(\mathbb{Z}) .
$$

Thus we have a natural map

$$
\mathrm{O}_{n}(\mathbb{Z}) \star_{\mathrm{O}_{4}(\mathbb{Z}) \times \mathrm{O}_{n-4}(\mathbb{Z})} \operatorname{Aut}\left(\mathrm{D}_{4}\right) \times \mathrm{O}_{n-4}(\mathbb{Z}) \rightarrow \mathrm{O}_{n}\left(\mathbb{Z}\left[\frac{1}{2}\right]\right)
$$

which is onto if $Y_{n}$ is connected. Since $\mathrm{O}_{n}(\mathbb{Z})$ and $\operatorname{Aut}\left(\mathrm{D}_{4}\right)$ are both generated by reflections the result follows from the following considerations. 
For $n \leq 7$, the graph $Y_{n}$ is nothing but the graph considered by M. Kneser in [K1957], so it is connected (see loc. cit.).

For $n \geq 8$, we prove by induction on $n$ that $Y_{n}$ is connected. Thus assume $Y_{n-1}$ is connected. Let us pick two lattices $L$ and $L^{\prime}$ that are isometric with $\mathrm{I}_{n}(\mathbb{Z})$ with respective orthonormal bases $\left(b_{1}, \ldots, b_{n}\right)$ and $\left(b_{1}^{\prime}, \ldots, b_{n}^{\prime}\right)$. Then by corollary 21 there exists a unitary vector $f$ that is both orthogonal to $b_{1}$ and $b_{1}^{\prime}$. Let $K$ (resp. $K^{\prime}$ ) be a lattice generated by an orthonormal basis beginning with $\left(b_{1}, c\right)$ (resp. $\left(b_{1}^{\prime}, c\right)$ ). By using the induction hypothesis in $b_{1}^{\perp}$ (resp. in $\left(b_{1}^{\prime}\right)^{\perp}$, resp. in $c^{\perp}$ ), we see that there exists a path from $L$ to $K$ (resp. from $L^{\prime}$ to $K^{\prime}$, resp. from $K$ to $\left.K^{\prime}\right)$.

\subsubsection{The equality $\left[\mathrm{O}_{n}\left(\mathbb{Z}\left[\frac{1}{2}\right]\right), \mathrm{O}_{n}\left(\mathbb{Z}\left[\frac{1}{2}\right]\right)\right]=\mathrm{SO}_{n}^{\prime}\left(\mathbb{Z}\left[\frac{1}{2}\right]\right)(n \geq 1)$.}

Recall that $\mathrm{SO}_{n}^{\prime}\left(\mathbb{Z}\left[\frac{1}{2}\right]\right)$ is the subgroup of $\mathrm{O}_{n}\left(\mathbb{Z}\left[\frac{1}{2}\right]\right)$ whose elements are products $\tau_{u_{1}} \circ \cdots \circ \tau_{u_{s}}$ of reflections

$$
\tau_{u_{k}}: x \mapsto x-2 \frac{\left(x, u_{k}\right)}{q\left(u_{k}\right)} u_{k}
$$

with $s$ even and $\prod_{k=1}^{s} q\left(u_{k}\right) \in \mathbb{Z}\left[\frac{1}{2}\right]^{\times 2}$.

Clearly, there is an inclusion $\left[\mathrm{O}_{n}\left(\mathbb{Z}\left[\frac{1}{2}\right]\right), \mathrm{O}_{n}\left(\mathbb{Z}\left[\frac{1}{2}\right]\right)\right] \subset \mathrm{SO}_{n}^{\prime}\left(\mathbb{Z}\left[\frac{1}{2}\right]\right)$. The other inclusion follows, as in [Dieudonné p.72], from the fact that in the decomposition $\tau_{u_{1}} \circ \cdots \circ \tau_{u_{s}}$ of an element of $\mathrm{SO}_{n}^{\prime}\left(\mathbb{Z}\left[\frac{1}{2}\right]\right)$, each $u_{k}$ may be chosen to have norm $q\left(u_{k}\right)=1$ or $q\left(u_{k}\right)=2$, i.e. to be in the orbit of one of the vectors $e_{1}$ or $e_{1}+e_{2}$ of $\mathrm{I}_{2}(\mathbb{Z})$.

\subsubsection{The equality $\left[\mathrm{SO}_{n}^{\prime}\left(\mathbb{Z}\left[\frac{1}{2}\right]\right), \mathrm{SO}_{n}^{\prime}\left(\mathbb{Z}\left[\frac{1}{2}\right]\right)\right]=\mathrm{SO}_{n}^{\prime}\left(\mathbb{Z}\left[\frac{1}{2}\right]\right)(n \geq 8)$.}

Only the inclusion $\mathrm{SO}_{n}^{\prime}\left(\mathbb{Z}\left[\frac{1}{2}\right]\right) \subset\left[\mathrm{SO}_{n}^{\prime}\left(\mathbb{Z}\left[\frac{1}{2}\right]\right), \mathrm{SO}_{n}^{\prime}\left(\mathbb{Z}\left[\frac{1}{2}\right]\right)\right]$ needs to be proved. First we note that the equality $\mathrm{SO}_{n}^{\prime}\left(\mathbb{Z}\left[\frac{1}{2}\right]\right)=\left[\mathrm{O}_{n}\left(\mathbb{Z}\left[\frac{1}{2}\right]\right), \mathrm{O}_{n}\left(\mathbb{Z}\left[\frac{1}{2}\right]\right)\right]$ and the fact that the set of reflections is closed under conjugation (by elements of $\left.\mathrm{O}_{n}\left(\mathbb{Z}\left[\frac{1}{2}\right]\right)\right)$ shows that $\mathrm{SO}_{n}^{\prime}\left(\mathbb{Z}\left[\frac{1}{2}\right]\right)$ is generated by the commutators of reflections. Now if $\tau_{u_{1}} \tau_{u_{2}} \tau_{u_{1}} \tau_{u_{2}}$ is an element of $\mathrm{SO}_{n}^{\prime}\left(\mathbb{Z}\left[\frac{1}{2}\right]\right)$, then by proposition 22 there exists two vectors $x$ and $y$ in $\operatorname{span}\left(u_{1}, u_{2}\right)^{\perp}$ such that

$$
q(x)=q\left(u_{1}\right) \quad, \quad q(y)=q\left(u_{2}\right) \quad, \quad x \perp y .
$$

Finally, the equalities $\tau_{u_{1}-x}(x)=u_{1}$ and $\tau_{u_{2}-y}(y)=u_{2}$ furnish the identity

$$
\tau_{u_{1}} \tau_{u_{2}} \tau_{u_{1}} \tau_{u_{2}}=\left[\tau_{u_{1}-x} \tau_{x} \tau_{u_{1}-x} \tau_{x}, \tau_{u_{2}-y} \tau_{y} \tau_{u_{2}-y} \tau_{y}\right]
$$

3.3. On the homotopy groups of $\mathrm{BO}_{\infty}^{+}\left(\mathbb{Z}\left[\frac{1}{2}\right]\right)$. Let $\Gamma$ be a group with commutator subgroup $\Gamma_{0}$. Recall from [Rosenberg] that if $\Gamma_{0}$ is perfect, then it admits a (unique) universal central extension

$$
0 \rightarrow S \rightarrow \tilde{\Gamma}_{0} \rightarrow \Gamma_{0} \rightarrow 1
$$

where the abelian group $S$ is called the Schur multiplier of $\Gamma_{0}$, and we have

a) $\pi_{1}\left(\mathrm{~B} \Gamma^{+}\right)=\Gamma / \Gamma_{0}=\mathrm{H}_{1}(\Gamma ; \mathbb{Z})$ and $\mathrm{H}_{1}\left(\Gamma_{0} ; \mathbb{Z}\right)=0$

b) $\pi_{2}\left(\mathrm{~B} \Gamma^{+}\right)=\mathrm{H}_{2}\left(\Gamma_{0} ; \mathbb{Z}\right)=S$ and $\mathrm{H}_{2}\left(\tilde{\Gamma}_{0} ; \mathbb{Z}\right)=0$;

c) $\pi_{3}\left(\mathrm{~B} \Gamma^{+}\right)=\mathrm{H}_{3}\left(\tilde{\Gamma}_{0} ; \mathbb{Z}\right)$;

d) $\mathrm{H}_{n}\left(\mathrm{~B} \Gamma^{+}\right)=\mathrm{H}_{n}(\Gamma)$ for all $n$.

In our case, it follows from a) that there is an isomorphism $\pi_{1}\left(\mathrm{BO}_{\infty}^{+}\right) \stackrel{\operatorname{det}_{\theta}}{\longrightarrow} \mathbb{Z} / 2 \times \mathbb{Z} / 2$. 
3.3.1. Computation of $\pi_{2}\left(B O_{\infty}^{+}\left(\mathbb{Z}\left[\frac{1}{2}\right]\right)\right)$.

Proposition 24. The Schur multiplier $\mathrm{H}_{2}\left(\mathrm{SO}_{8}^{\prime}\left(\mathbb{Z}\left[\frac{1}{2}\right]\right)\right)$ is isomorphic to $\mathbb{Z} / 2$.

Proof: First the isomorphism $\mathrm{H}_{*}\left(\mathrm{BSO}_{8}^{\prime}\left(\mathbb{Z}\left[\frac{1}{2}\right]\right), \mathbb{Z}_{(2)}\right) \rightarrow \mathrm{H}_{*}\left(\mathrm{BSO}_{8}^{\prime}(\mathbb{Z} / 3), \mathbb{Z}_{(2)}\right)$ due to Henn and Lannes, the isomorphism $\mathrm{H}_{2}\left(\mathrm{BSO}_{8}^{\prime}(\mathbb{Z} / 3), \mathbb{Z}\right) \simeq \mathbb{Z} / 2$ (see the ATLAS, p. xvi), the nullity of $\mathrm{H}_{1}\left(\mathrm{BSO}_{8}^{\prime}\left(\mathbb{Z}\left[\frac{1}{2}\right]\right)\right.$ ), and the universal coefficients theorem, show that $\mathrm{H}_{2}\left(\mathrm{BSO}_{8}^{\prime}\left(\mathbb{Z}\left[\frac{1}{2}\right]\right) ; \mathbb{Z}_{(2)}\right) \simeq \mathbb{Z} / 2$. Thus it suffices to prove that $\mathrm{H}_{2}\left(\mathrm{BSO}_{8}^{\prime}\left(\mathbb{Z}\left[\frac{1}{2}\right]\right) ; \mathbb{Z}\left[\frac{1}{2}\right]\right)$ is trivial. In order to do so, we need to use a nice space acted upon by $\mathrm{SO}_{8}^{\prime}\left(\mathbb{Z}\left[\frac{1}{2}\right]\right)$. Such a space has been studied in detail by W. Frisch $[\mathrm{F}]$.

$\circ$ Let $\mathrm{D}_{8} \subset \mathrm{I}_{8}\left(\mathbb{Z}\left[\frac{1}{2}\right]\right)$ be the $\mathbb{Z}$-sublattice of index 2 in $\mathrm{I}_{8}(\mathbb{Z})$ whose vectors $x$ that satisfy the congruence $x_{1}+\cdots+x_{8} \equiv 0(\bmod .2)$.

$\circ$ Put $v^{ \pm}=\frac{e_{1}+\cdots+e_{7} \pm e_{8}}{2}$. Then $\mathrm{D}_{8}$ is contained in $\left(\mathrm{I}_{8}(\mathbb{Z})\right.$ and $)$ the two even unimodular lattices $\mathrm{E}_{8}^{ \pm}=$ $\mathrm{D}_{8}+\mathbb{Z} v^{ \pm}$.

- Let $\mathrm{D}_{4} \perp \mathrm{D}_{4}$ be the $\mathbb{Z}$-sublattice of index 2 in $\mathrm{D}_{8}$ consisting of vectors $x$ that satisfy the congruence $x_{1}+\cdots+x_{4} \equiv 0(\bmod .2)$.

○ Let ' $\mathrm{D}_{8}$ be the sublattice of index 2 in $\mathrm{D}_{4} \perp \mathrm{D}_{4}$ consisting of vectors $x$ that satisfy the congruence $x_{3}+x_{4}+x_{5}+x_{6} \equiv 0(\bmod .2)$. Note that there exists an isomorphism ${ }^{\prime} \mathrm{D}_{8} \simeq 2 \otimes\left(\mathrm{D}_{8}^{\sharp}\right)$.

○ Let ' $\mathrm{E}_{8}^{+}$be the sublattice of index 2 in ' $\mathrm{D}_{8}$ consisting of vectors $x$ that satisfy the congruence $x_{1}+x_{4}+$ $x_{5}+x_{8} \equiv 0(\bmod .2)$. Note that there exists an isomorphism ' $\mathrm{E}_{8}^{+} \simeq 2 \otimes\left(\mathrm{E}_{8}^{ \pm \sharp}\right)$. Similarly, let ' $\mathrm{E}_{8}^{-}$be the sublattice of index 2 in ' $D_{8}$ consisting of vectors $x$ that satisfy the congruence $x_{1}+x_{3}+x_{6}+x_{7} \equiv 0(\bmod .2)$. Once again there exists an isomorphism ' $\mathrm{E}_{8}^{-} \simeq 2 \otimes\left(\mathrm{E}_{8}^{ \pm \sharp}\right)$.

Let $\mathcal{O}$ be the geometric realization of the simplicial set whose set of vertices is the set $K$ of $\mathbb{Z}$-lattices in $\mathrm{I}_{8}\left(\mathbb{Z}\left[\frac{1}{2}\right]\right)$ that are isometric to $\mathrm{E}_{8}^{ \pm}$or ' $\mathrm{E}_{8}^{ \pm}$, and whose set of maximal vertices are subsets $\left\{L_{a}, L_{b}, L_{c}, L_{d}\right\}$ for which the following properties hold

$$
L_{a} \simeq{ }^{\prime} \mathrm{E}_{8}^{ \pm} \simeq L_{b}, \quad L_{c} \simeq \mathrm{E}_{8}^{ \pm} \simeq L_{d},{ }^{\prime} \mathrm{D}_{8} \simeq L_{a}+L_{b} \subset L_{c} \cap L_{d} \simeq \mathrm{D}_{8} .
$$

It follows almost directly from $[\mathrm{F}]$

$\circ$ that $\mathcal{O}$ is 3 -acyclic,

○ that if an element of $\mathrm{SO}_{8}^{\prime}\left(\mathbb{Z}\left[\frac{1}{2}\right]\right)$ stabilizes globally a simplex of $\mathcal{O}$, it stabilizes it pointwise,

○ that the action of $\mathrm{SO}_{8}^{\prime}\left(\mathbb{Z}\left[\frac{1}{2}\right]\right)$ is transitive on maximal simplices of $\mathcal{O}$,

o that the stabilizer of any vertex is isomorphic to an extension of $\mathrm{SO}_{8}^{\prime}\left(\mathbb{F}_{2}\right)$ by an elementary abelian 2group (in particular has trivial $\mathrm{H}_{1}\left(-; \mathbb{Z}\left[\frac{1}{2}\right]\right)$ since $\mathrm{SO}_{8}^{\prime}\left(\mathbb{F}_{2}\right)$ is simple, and trivial $\mathrm{H}_{2}\left(-; \mathbb{Z}\left[\frac{1}{2}\right]\right)$ since the Schur multiplier of $\mathrm{SO}_{8}^{\prime}\left(\mathbb{F}_{2}\right)$ is 2-torsion (see [ATLAS, p. xvi])).

o that the stabilizer of the edges $\left({ }^{-} \mathrm{E}_{8}^{-}, \mathrm{E}_{8}^{+}\right)$and $\left(\mathrm{E}_{8}^{-}, \mathrm{E}_{8}^{+}\right)$are isomorphic to an extension of the alternating group $\mathfrak{A}_{8}$ by an elementary abelian 2-group, (in particular have trivial $\mathrm{H}_{1}\left(-; \mathbb{Z}\left[\frac{1}{2}\right]\right)$ ).

o that the stabilizer of the edges $\left(' \mathrm{E}_{8}^{ \pm}, \mathrm{E}_{8}^{ \pm}\right)$are isomorphic to an extension of the group $\mathrm{SL}_{4}\left(\mathbb{F}_{2}\right)$ by an elementary abelian 2-group (in particular have trivial $\mathrm{H}_{1}\left(-; \mathbb{Z}\left[\frac{1}{2}\right]\right)$ ).

By injecting these pieces of information into the stabilizer spectral sequence (see [Brown Chap. VII]), we obtain the triviality of $\left.\mathrm{H}_{2}\left(\mathrm{SO}_{8}^{\prime}\left(\mathbb{Z}\left[\frac{1}{2}\right]\right), \mathbb{Z}\left[\frac{1}{2}\right]\right)\right)$.

Corollary 25. For $n \geq 8$, the Schur multiplier of $\mathrm{SO}_{n}^{\prime}\left(\mathbb{Z}\left[\frac{1}{2}\right]\right)$ is isomorphic to $\mathbb{Z} / 2$.

Proof: The action of $\mathrm{SO}_{n}^{\prime}\left(\mathbb{Z}\left[\frac{1}{2}\right]\right)$ on the space $X_{n}$ is transitive on $k$-simplices for $k \leq 2$. Since $\mathrm{H}_{1}\left(\mathrm{SO}_{n}^{\prime}\left(\mathbb{Z}\left[\frac{1}{2}\right]\right)\right)$ is trivial for $n \geq 7$, it follows that the natural morphism $\mathrm{H}_{2}\left(\mathrm{SO}_{n}^{\prime}\left(\mathbb{Z}\left[\frac{1}{2}\right]\right)\right) \rightarrow \mathrm{H}_{2}\left(\mathrm{SO}_{n+1}^{\prime}\left(\mathbb{Z}\left[\frac{1}{2}\right]\right)\right)$ is onto. Since the Spin extension is central and non-trivial, $\mathrm{H}_{2}\left(\mathrm{SO}_{n+1}^{\prime}\left(\mathbb{Z}\left[\frac{1}{2}\right]\right)\right)$ projects onto $\mathbb{Z} / 2$. 


\subsubsection{Computation of $\pi_{3}\left(B O_{\infty}^{+}\left(\mathbb{Z}\left[\frac{1}{2}\right]\right)\right) \otimes \mathbb{Z}_{(2)}$.}

Let $R$ be either $\mathbb{Z}\left[\frac{1}{2}\right]$ or $\mathbb{Z} / 3$. From the fact that the action of $\mathrm{SO}_{n}^{\prime}(R)$ on the space $X_{n}(R)$ is transitive on $k$-simplices for $k \leq n-2$, that $\mathrm{H}_{1}\left(\operatorname{Spin}_{n}(R)\right)$ and $\mathrm{H}_{2}\left(\operatorname{Spin}_{n}(R)\right)$ are trivial for $n \geq 8$, and that $X_{n}$ is 2 -acyclic for $n \geq 15$, it follows that $\mathrm{H}_{3}\left(\operatorname{Spin}_{n}(R)\right) \rightarrow \mathrm{H}_{3}\left(\operatorname{Spin}_{n+1}(R)\right)$ is an isomorphism for $n \geq 14$. Now on one hand, Henn and Lannes proved that the natural map $\mathrm{H}_{*}\left(\operatorname{Spin}_{14}\left(\mathbb{Z}\left[\frac{1}{2}\right]\right) ; \mathbb{Z}_{(2)}\right) \rightarrow \mathrm{H}_{*}\left(\operatorname{Spin}_{14}(\mathbb{Z} / 3) ; \mathbb{Z}_{(2)}\right)$ is an isomorphism, and on the other hand, [Fiedorowicz-Priddy] have computed $\pi_{3}\left(\mathrm{BO}_{\infty}^{+}(\mathbb{Z} / 3)\right) \otimes \mathbb{Z}_{(2)}=\mathbb{Z} / 8$. Summarizing, we obtain isomorphisms

$$
\begin{array}{ccc}
\mathrm{H}_{3}\left(\operatorname{Spin}_{14}\left(\mathbb{Z}\left[\frac{1}{2}\right]\right), \mathbb{Z}_{(2)}\right) & =\mathrm{H}_{3}\left(\operatorname{Spin}_{14}(\mathbb{Z} / 3), \mathbb{Z}_{(2)}\right)= & \left.\pi_{3}\left(\mathrm{BO}_{\infty}^{+}(\mathbb{Z} / 3)\right) \otimes \mathbb{Z}_{(2)}\right) \\
\pi_{3}\left(\mathrm{BO}_{\infty}^{+}\left(\mathbb{Z}\left[\frac{1}{2}\right]\right)\right) \otimes \mathbb{Z}_{(2)} & \| \\
\mathbb{Z} / 8
\end{array} .
$$

\section{REFERENCES}

Brown. K.S. Brown ; Cohomology of Groups. GTM 87, Springer Verlag, 1982.

Ca2007. J.-L. Cathelineau; Homology stability for orthogonal groups over algebraically closed fields. Ann. Sc. E.N.S. 40(3), 487-517, 2007.

Ch1979. R. M. Charney ; Homology stability for $\mathrm{GL}_{n}$ over Dedekind domains. Bull. A.M.S. 1(2), 428-431, 1979.

ATLAS. J. H. Conway, R. A. Parker, S. P. Norton ; The ATLAS of finite groups, 1985.

Dieudonné. J. Dieudonné ; La géométrie des groupes classiques. Ergebnisse der Math. 5, Springer Verlag 1971.

Fiedorowicz-Priddy. Z. Fiedorowicz, S. Priddy ; Homology of classical groups over finite fields and their associated infinite loop spaces, LNM. 674, Springer Verlag, 1978.

F. W. Frisch ; Thesis, available at

http://www.uni-math.gwdg.de/preprint/meta/mg.2003.08.html.

HW. A. Hatcher, N. Wahl ; Stabilization for mapping class groups of 3-Manifolds, preprint available at http://arxiv.org/0709.2173v3.

HL. H.-W. Henn, J. Lannes ; in preparation.

J1962. R. Jacobowitz ; Hermitian forms over local fields, Amer. J. Math. 84, 441-465, 1962.

K1957. M. Kneser ; Klassenzahlen definiter quadratischer Formen. Arch. Math. 8, 241-250, 1957.

K1966. M. Kneser ; Strong Approximation. In Algebraic groups and discontinuous subgroups, Proc. Symp. Pure Math. 9, AMS, 1966.

MvdK2002. B. Mizraii, W. van der Kallen ; Homology stability for unitary groups. Doc. Math. 7, 143-166, 2002.

O'Meara. O.T. O'Meara ; Introduction to quadratic forms. Grundlehren der math. 117, Springer Verlag 1963.

Rosenberg. J. Rosenberg ; Algebraic K-theory and its applications. GTM 147, Springer Verlag, 1994.

S1964. G. Shimura, Arithmetic of unitary groups, Annals of Math. 79, 369-409, 1964.

vdK1980. W. van der Kallen; Homology stability for linear groups. Invent. Math. 60(3), 269-295, 1980.

V1977. K. Vogtmann, Thesis, University of California, 1977.

V1982. K. Vogtmann, A Stiefel Complex for the orthogonal group of a field, Comment. Math. Helv. $\mathbf{5 7}(1), 11-21,1982$.

Institut de Recherche Mathématiques Avancées UMR 7501 de l'Université de Strasbourg et du CNRS.

email: gael.collinet@math.unistra.fr

Received: January 7, 2009. 